\title{
Molecularly imprinted polymers in biological applications
}

Zahra El-Schich ${ }^{1}$ (D), Yuecheng Zhang ${ }^{1,2}$, Marek Feith ${ }^{1,3}$ (D), Sarah Beyer ${ }^{1,2}$, Louise Sternbæk ${ }^{1,2,4}$, Lars Ohlsson ${ }^{1,2}$, Maria Stollenwerk ${ }^{1,2}$ \& Anette Gjörloff Wingren*,1,2 (iD

${ }^{1}$ Department of Biomedical Sciences, Faculty of Health \& Society, Malmö University, Malmö, Sweden; ${ }^{2}$ Biofilms-Research Center for Biointerfaces, Malmö University, Malmö, Sweden; ${ }^{3}$ Department of Physiology, Faculty of Medicine, Masaryk University, Brno, Czech Republic; ${ }^{4}$ Phase Holographic Imaging AB, Lund, Sweden; *Author for correspondence: anette.gjorloff-wingren@mau.se

BioTechniques 69: 407-419 (December 2020) 10.2144/btn-2020-0091

First draft submitted: 24 June 2020; Accepted for publication: 21 August 2020; Published online: 1 October 2020

\section{ABSTRACT}

Molecularly imprinted polymers (MIPs) are currently widely used and further developed for biological applications. The MIP synthesis procedure is a key process, and a wide variety of protocols exist. The templates that are used for imprinting vary from the smallest glycosylated glycan structures or even amino acids to whole proteins or bacteria. The low cost, quick preparation, stability and reproducibility have been highlighted as advantages of MIPs. The biological applications utilizing MIPs discussed here include enzyme-linked assays, sensors, in vivo applications, drug delivery, cancer diagnostics and more. Indeed, there are numerous examples of how MIPs can be used as recognition elements similar to natural antibodies.

Relevant biomarkers are urgently needed to be able to improve diagnosis, prognosis and treatment of diseases. Biomarkers are often a gene, RNA, a protein or the combination thereof. For early diagnosis of a disease, the biomarker should be part of a routine screening examination or at the first sign of a questionable symptom [1]. To detect the biomarker, the proper methods and tools need to be selected.

Antibodies, the natural receptors for proteins and peptides, have been the most widely used analytical tool in clinical settings for the detection of those molecules. However, antibodies are not the best choice for targeting small molecules with a lower degree of complexity, such as lipids and carbohydrates, due to low affinity and specificity of the resulting antibody. Therefore, targeting carbohydrates require other tools, such as lectins or molecularly imprinted polymers (MIPs). Lectins can contribute to clinical diagnostics by the binding to glycoconjugates on cells [2]. The design of MIPs and their use as artificial recognition elements have shown to be successful for targeting both glycans and other molecules, as we will focus on in this review. The target molecule, the template, can in fact be a protein, peptide, lipid, amino acid, virus, a cell, nucleic acid or even more complex glycan structures (Figure 1).

The technique was first reported in 1972 by Wulff and Sarhan [3], and molecular imprinting has since then been adopted by many research groups, as evidenced by the increase in studies over the past years. The production of MIPs is reproducible, quick, cheap, stable and economical, but challenges still exist. Besides the choice of a proper template and preparation thereof, initiators, solvent compositions, functional monomers and cross-linkers must be selected carefully and the quality of the final MIPs tested thoroughly. The biological applications of MIPs are extensive: assays such as ELISA and sensors, imaging and in vivo applications including drug delivery, inhibition of cell signaling and more.

In an excellent way, recent reviews have already discussed some of the main perspectives and applications of strategic MIP synthesis [4-7]. Moreover, MIP-based imaging and selection of the core material was discussed by Vaneckova et al. [8]. Whole cells and cell epitope development for MIP imprinting have been highlighted recently $[9,10]$, as well as MIP-based electrochemical and optical sensors [11,12]. Finally, Xu et al. evaluated the advantages, and pointed out the shortcomings of the use of MIPs in biomedical applications today, to inspire the future development of the field [13]. In this review, we aim to summarize different imprinting strategies together with the most recent biomedical applications of MIPs in cancer diagnostics of humans and animals.

\section{MIIPs \& synthesis}

The technique of molecular imprinting can be described by a technology of creating artificial tailor-made binding sites that are complementary to the size, shape and functional groups of the template molecule. Sialic acid (SA) is used as a proof of concept molecule to illustrate the principle of the surface-imprinting coupled with reversible addition fragmentation chain transfer polymerization and the selectivity of the MIPs toward SA on the surface of cancer cells (Figure 2). The synthesis procedure is a key process that directs the MIP properties. Generally, the mechanism of MIP formation is broadly classified into a free-radical polymerization and sol-gel process, where the former one is more general.

An overview of polymerization techniques is shown in Table 1. Among the free-radical polymerization methods, bulk polymerization is a well-used method based on its attractive properties, including rapidity, simplicity and no need for sophisticated instruments [14]. The synthesized bulk polymer monolith has to be crushed, grounded and sieved to an appropriate size, which is time consuming [15]. This 


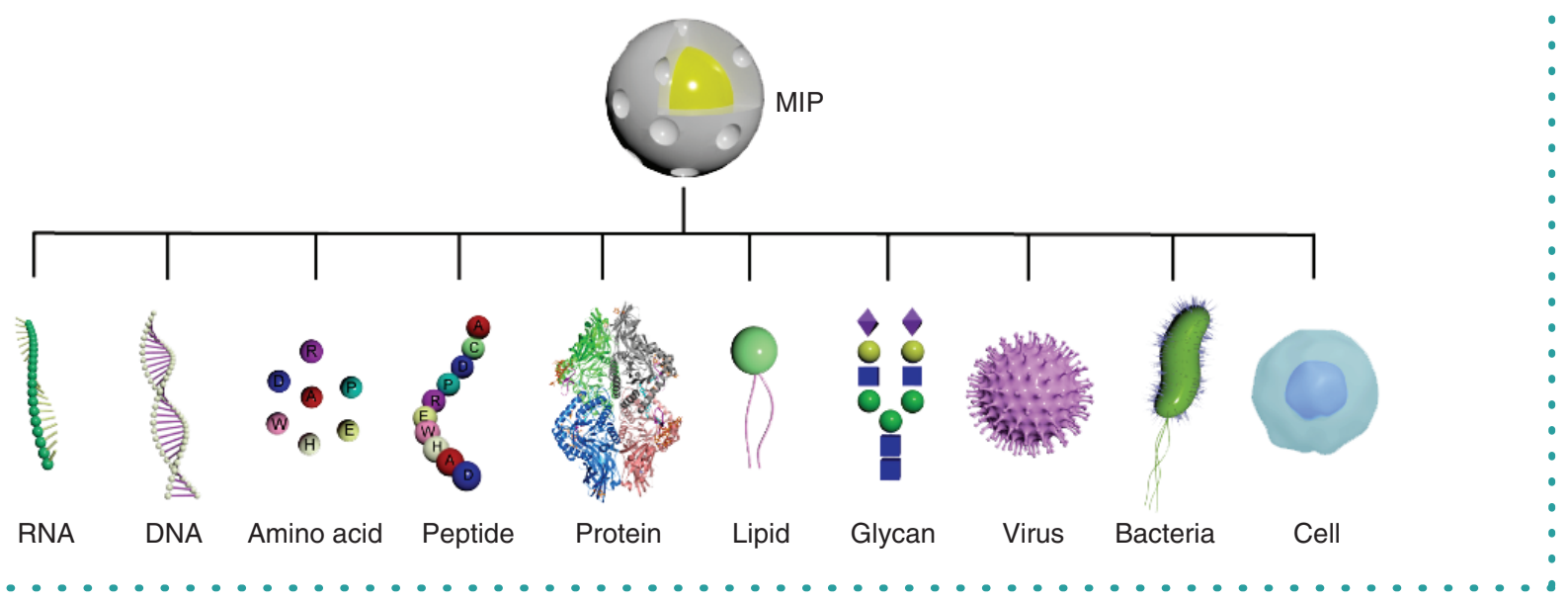

Figure 1. The templates for imprinting the polymer particles - molecularly imprinted polymers - are numerous. From left to right, templates such as RNA, DNA, amino acids, peptide, protein, lipid, glycan, virus, bacteria and a cell are shown.

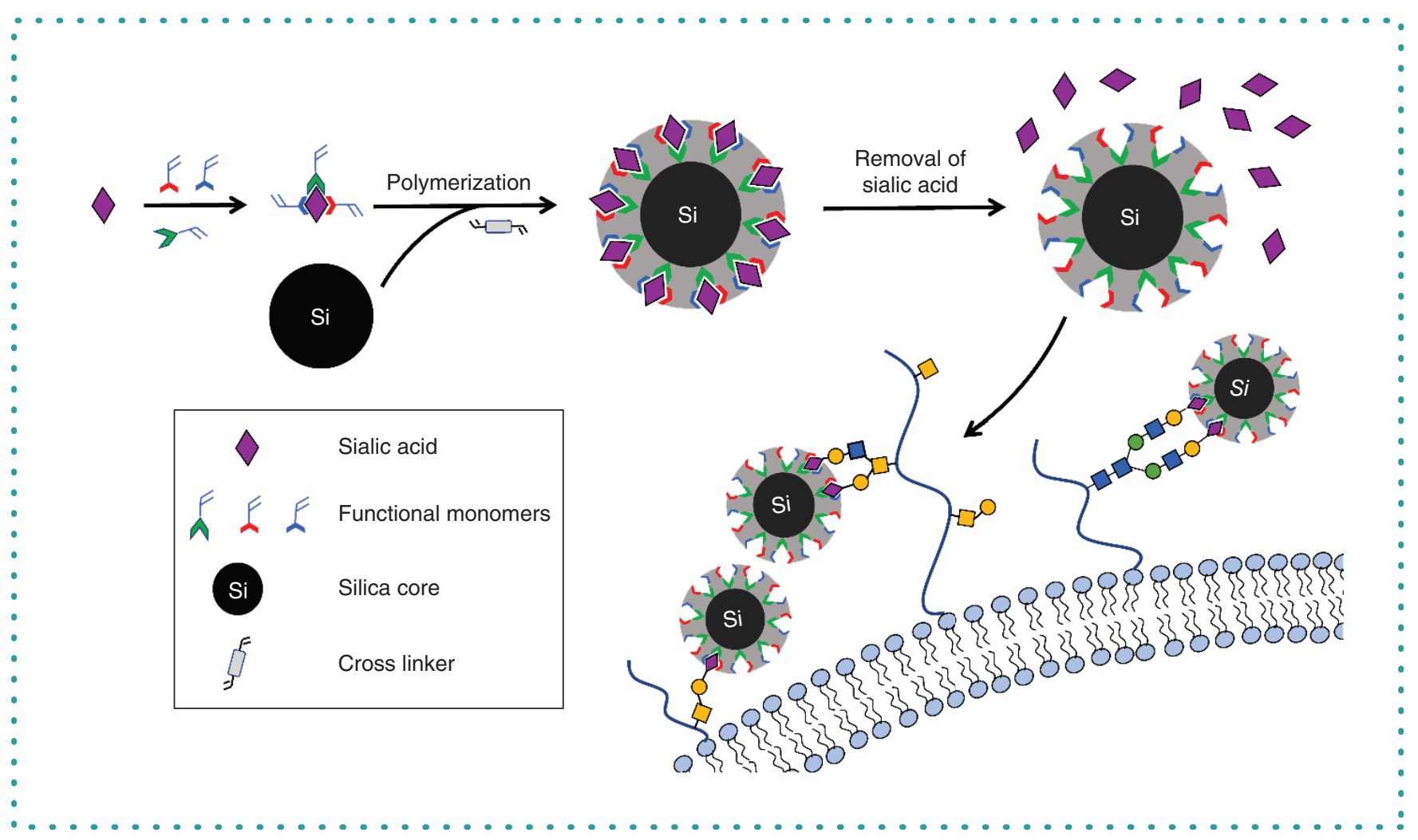

Figure 2. Schematic illustration of the molecularly imprinted polymer synthesis mechanism and its selectivity toward sialic acid on cancer cells.

method requires a large amount of template [16], and some high-affinity binding sites are destroyed during this process. To overcome these drawbacks, a variety of other polymerization techniques have been developed, such as suspension polymerization, emulsion polymerization, seed polymerization and precipitation polymerization.

The suspension polymerization method is another common method used for MIP synthesis. It provides a heterogeneous polymerization environment where water [47], perfluorocarbon liquid [48] or mineral oil [49] can be used as a continuous phase to suspend the droplet of the pre-polymerization mixture. Each droplet reacts like a mini bulk reaction that can produce the spherical MIP particles with a broad size range [14]. 


\begin{tabular}{|c|c|c|}
\hline Imprinting methods & Examples of templates & Ref. \\
\hline Bulk polymerization & Iridoid glycosides, spiramycin, 2,4-dichlorophenoxyacetic acid, diclofenac, alfatoxins & [17-21] \\
\hline Suspension polymerization & Malachite green, p-hydroxybenzoic acid, timolol, alfatoxins $\mathrm{B} 1, \mathrm{~Pb}^{2+}$ & [22-26] \\
\hline Emulsion polymerization & Quercetin, malachite green, Listeria monocytogenes, triazines, $\mathrm{Cd}^{2+}$ & [27-31] \\
\hline Seed polymerization & Nicotinamide, bisphenol A, promazine derivates & [32-34] \\
\hline Precipitation polymerization & Triclosan and triclocarban, strychnine, caffeic acid, vitamin E, prednisolone & [35-39] \\
\hline RAFT polymerization & Benzimidazole, propranolol, polycyclic aromatic hydrocarbons & [40-42] \\
\hline Atom transfer radical polymerization & Ofloxacin, $\beta 2$-agonists & {$[43,44]$} \\
\hline Sol-gel & Iprodione, methylxanthines & {$[45,46]$} \\
\hline
\end{tabular}

Emulsion polymerization and seed polymerization can both produce monodispersed MIP particles. The MIPs produced by emulsion polymerization are suffering from the presence of remnants of surfactant [50], whereas the seed polymerization needs a time-consuming multistep procedure [51].

Precipitation polymerization is a single preparative step method that can produce MIP microspheres with a relatively uniform size. Due to the simplicity and excellent size control ability, this method has become one of the most promising approaches to produce highquality spherical imprinted particles [14]. In the polymerization process, the polymer chain can grow individually in the diluted solution, becoming insoluble and precipitate from the solution [52]. The size and shape of the MIPs can be well controlled by the strict reaction conditions, including the polarity of the solvents [53], polymerization temperature [54] and stirring speed [55]. The drawbacks of this method are the need of large volumes of porogen and a long polymerization time.

Besides these conventional polymerization methods, many new techniques have been promoted to synthesize MIPs in recent years, including reversible addition fragmentation chain transfer [56], nitroxide-mediated polymerization [57] and metal-catalyzed atom transfer radical polymerization [58]. Compared with the conventional methods, these methods involve a thermodynamically controlled process that can slow the rate for the polymer chain growth and therefore produce more homogeneous polymers with a narrow distribution of polymer chain length.

The sol-gel process is another convenient and versatile method in MIP synthesis, which produces a transparent sol-gel matrix to capture numerous organic, organometallic and biological molecules [59,60]. In the general sol-gel process, a colloidal solution (sol) is poly-condensed into a solid (gel) phase. This process has several advantages, one of which is the use of the silica-based materials that can form a rigid high degree cross-linking $\left(\mathrm{SiO}_{2}\right)_{\mathrm{n}}$ network, which can retain the shape of the cavities after the removal of the template. Moreover, this method uses eco-friendly solvents, such as water, and it can easily be produced at room temperature [61].

\section{Surface-imprinted synthesis}

More recently, an increasing number of imprinting procedures were accrued on the surface of particles. Compared with the traditional MIPs, surface-imprinted polymers (SIPs) exhibit advantages not only including higher binding capacity and faster mass transfer but also merits shared by the particles themselves. Many particles have been used to synthesize SIP particles, including activated silica gel [56,62,63], $\mathrm{Fe}_{3} \mathrm{O}_{4}$ magnetic nanoparticles [64,65], quantum dots (QDs) [66,67] and carbon QDs [68,69]. Among them, silica gel is a widely used particle in the surface-imprinting process due to its excellent characteristics, like high stability under acidic conditions and high thermal resistance. The silica gel-based surface-imprinted particles have been applied to metal ions [70,71], pharmaceuticals [72,73], glycans $[74,75]$ and proteins $[76,77]$ in the past few years. In addition, magnetic bead-based MIPs have been widely used for the separation of complicated samples due to their simplicity, high efficiency, low cost and remarkable advantages of magnetic separation [78].

\section{MIIPs \& biomarkers}

A biomarker shows a specific physical trait or a measurable biologically produced change in the body that is linked to a disease or a health condition [1]. Delays in results are still responsible for a significant number of mortalities and morbidities, and hence there is a need for faster, cost-effective and sensitive analytical methods. MIPs could help solve these issues, as MIPs are synthetic materials that can mimic biological recognition well enough to be called 'plastic antibodies' [79]. In the past decade, the number of studies toward using MIPs as a biomarker has increased, as they hold a potential to provide a sensitive, undamaged, rapid and low-cost diagnostic tool [80]. The detection of serum albumin [81,82], hemoglobin [83,84], ferritin [85] and avidin [86], as well as studies of infectious diseases [87-89], bone loss [90,91], cardiovascular diseases [92] and various cancers are some areas [4,93-102] that are of high interest for the use of MIPs as biomarkers.

MIPs target small molecules approximately ranging between $\sim 0.1$ and $\sim 10 \mathrm{~nm}$, whereas SIPs are applied for larger biomarkers [11]. SIPs can form cavities directly on the surface of polymers, which enhances the removal of the template, providing better use for larger biomarkers such as viruses, bacteria or cells. 


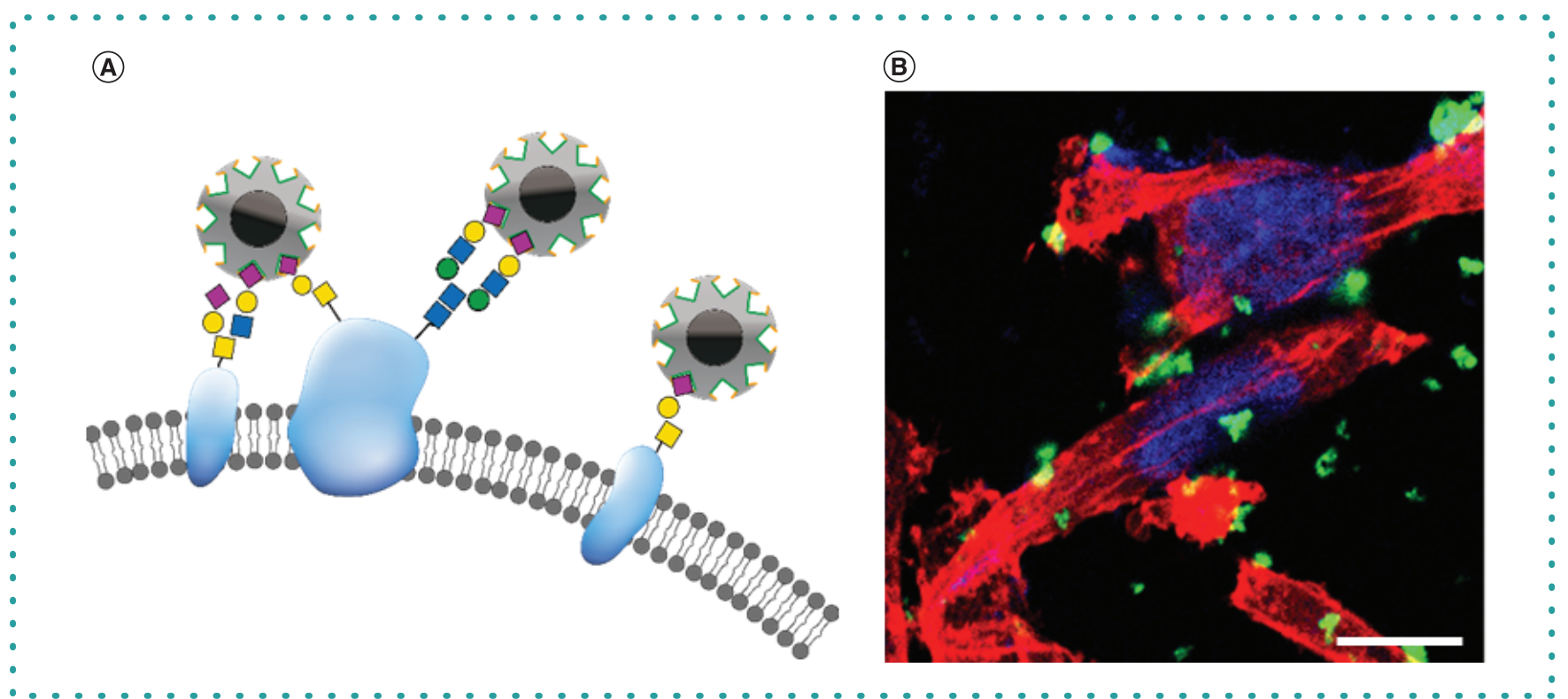

Figure 3. Molecularly imprinted polymers targeting sialic acid on the cell surface of a breast cancer cell line. (A) Schematic picture of sialic acid (SA) molecularly imprinted polymer binding to SA at the terminal end of the glycans at the surface of the cell membrane. (B) Fluorescent SA - molecularly imprinted polymers in a concentration of $0.1 \mathrm{mg} / \mathrm{ml}$ bind to the surface of the breast cancer cell line MDA-MB-231. The cytoskeleton is stained in red with phalloidin and the nuclei in blue with DAPI. Scale bar at $10 \mu \mathrm{m}$.

The development of protocols for the synthesis of MIPs targeting glycans and glycosaminoglycans (GAGs) have recently emerged due to the need for detection tools targeting carbohydrate structures. Since antibodies and lectins against glycans and GAGs are lacking both specificity and stability, and are expensive to produce, MIPs have been considered to be used as an alternative $[4,5,8-10,13,56,103,104]$.

Hyaluronan (HA) is a nonsulfated GAG and a major component of the extracellular matrix. The monosaccharide glucuronic acid, which is a part of HA, was used as a template for molecular imprinting [105]. Apart from the fact that a successful imprinting of glucuronic acid was shown, the authors also evaluated beneficial environmental protocols for the synthesis. Indeed, other approaches have been shown for MIP synthesis using glucuronic acid or SA as the template [94,106-111]. The SA-MIPs target SA at the terminal end of the glycosylated cell surface membrane proteins (Figure 3A). The glycan chains are usually branched and/or elongated. El-Schich ET AL. [SUBMITTED MANUSCRIPT (2020)] showed that fluorescent SA-MIPs synthesized according to the protocol of Shinde et al. [56] bind to MDA-MB-231 breast cancer cells, decorating the surface membrane of the cells (Figure 3B).

MIP-based sensors for point-of-care diagnostics is an increasing field, as MIPs can be combined with several different electrochemical transducers in a wide variety of sensor platforms [112]. Eersels et al. incorporated MIPs into a thermal biomimetic sensor platform for detection of vitamin $\mathrm{K}$ in serum samples [90]. UV-visible spectroscopy and two custom-made thermal readout techniques were used to analyze the target rebinding of a synthetic vitamin $\mathrm{K}$ compound, which was both selective and specific [90]. Serum C-terminal telopeptide of Type I collagen is a turnover biomarker for bone loss, and by combining MIP technology, both sensitive and rapid measurement of serum C-terminal telopeptide of Type I collagen could be performed [91].

A technique to determine cardiovascular biomarkers relies on expensive, time-consuming and complex procedures, which are unsuitable to carry out at the point of care. Moreira et al. used an alternative approach by using myoglobin-imprinted films by electropolymerizing o-aminophenol around a protein layer previously absorbed to gold. The myoglobin biosensor was evaluated by electrochemical impedance spectroscopy and square wave voltammetry. A good selectivity toward myoglobin was presented together with short measuring time, reusability and low limit of detection [92].

A major challenge in molecular imprinting is targeting of viruses due to their comparatively large dimensions (vs usually imprinted small molecules), fragile architecture and poor stability in organic solvents. Adenovirus-imprinted particles were synthesized providing enhanced selective virus recognition by coating with blocking agents such as polyethylene glycerol or bovine serum albumin to prevent unspecific binding [87]. Interestingly, a new 'double-imprinted' method was applied, based on a virus-bioimprinted hydrogel, which is micromolded into a diffraction-grating sensor to give a MIP gel laser diffraction sensor. The target in this study was apple stem pitting virus, which showed good selectivity and thereby confirms that the mechanism of the hydrogel volume change involves specific binding interactions between the apple stem pitting virus and the hydrogel-bound aptamers [88]. Although some successful virus imprints have already been developed, further progress is required regarding size of the target, its fragile structure, its functional complexity and its limited solubility. These challenges may be addressed by innovative imprinting strategies that enable utilizing virus-imprinted materials. This could be virus-imprinted materials that are packed into columns for selective/enrichment of viral species [89]. 


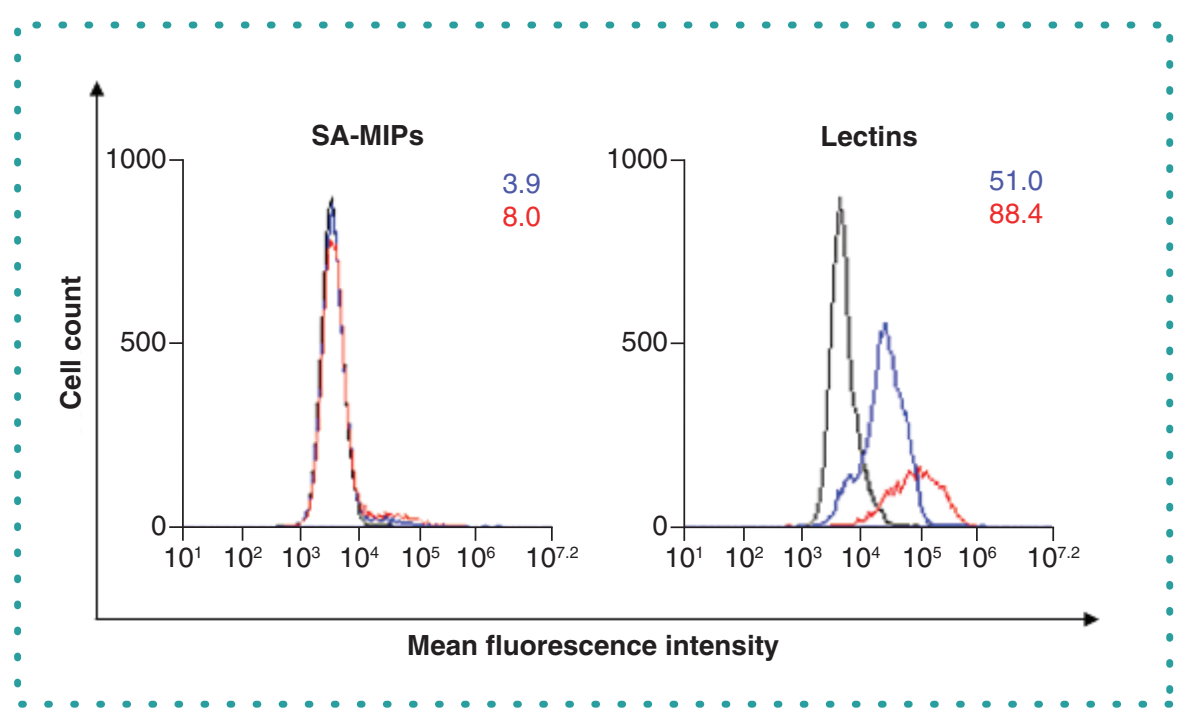

Figure 4. Histograms presenting the mean fluorescence values of the T47D breast cancer cell line stained with (left histogram) dSA-molecularly imprinted polymers in a concentration of $0.04 \mathrm{mg} / \mathrm{ml}$ (blue) and sialic acid - molecularly imprinted polymers in a concentration of $0.1 \mathrm{mg} / \mathrm{ml}$ (red), and (right histogram) lectins MAL (blue) and SNA (red) both in $5 \mu \mathrm{g} / \mathrm{ml}$. Numbers show the positivity of the cells in percentages as analyzed by flow cytometry.

Another target for a MIP-based biosensor is bacterial detection that is needed in many fields, including biosafety, clinical diagnostics, food safety and biosecurity. A study done by Golabi et al. used 3-aminophenylboronic acid for the electrochemical fabrication of cellimprinted polymers. Boronic acid groups were attached to the surface of Staphylococcus epidermidis, and electropolymerization was used to preserve the structure of the bacteria at the polymer network. Finally, the cells were removed, and the complementary cavities remained, some of which contained boronic acid for increased affinity. Compared with other bacteria, the MIPs showed high specificity and affinity for S. epidermidis [113]. Moreover, chitosan has been used to create MIP matrices that had high bacteria affinity [114].

Cancer biomarkers have become more compelling over the past decade because they provide early diagnostics, which increases survival. Pyruvic acid, SA, pro gastrin releasing peptide, p53, carbohydrate antigen 15-3, CEA, PSA, EGFR and VEGF are some of the cancer biomarkers that have been studied by using the molecular imprinting technique [4,93-102].

\section{MIIPs \& cancer}

Glycosylation has an important role in cancer biology and disease progression [115]. This makes SA an interesting biomarker for a diversity of cancer types. Several research groups have explored SA-imprinted MIPs in cancer-associated cellular applications $[56,94,103,104,106,107,110,111]$. Some normal epithelial cells express low amounts of SA, whereas malignant epithelial cells express more, a cellular system elegantly utilized by Wang et al. [104]. They showed that the hepatoma carcinoma HepG-2 and the breast carcinoma cell line MCF7 were exhibiting much stronger fluorescence with monosaccharide-imprinted nanoparticles, compared with normal hepatic cells and normal mammary epithelial cells MCF-10A. The monosaccharides investigated were SA, mannose and fucose [104]. Moreover, it was shown that SA-MIPs synthesized according to the protocol of Shinde et al. [56] bind efficiently to MCF7 cells (14.2\% as shown by flow cytometry analysis) [EL-SCHICH ET AL., SUBMITTED MANUSCRIPT (2020)]. Staining MCF7 cells with MAL and SNA lectins targeting 2,3-SA and 2,6-SA, respectively, it was shown that this invasive ductal breast carcinoma cell line expresses 2,3-SA but almost no 2,6-SA. Flow cytometry analysis of another invasive ductal breast carcinoma cell line, T47D, reveals that the same SA-MIPs bind to a lower degree ( $8 \%$ when using a concentration of $0.1 \mathrm{mg} / \mathrm{ml}$ of SA-MIPs and $3.9 \%$ when using a concentration of $0.04 \mathrm{mg} / \mathrm{ml}$ of dSA-MIPs) to this cell line (Figure 4). Interestingly, both 2,3-SA and 2,6-SA are expressed on T47D, according to the flow cytometry analysis using the lectins MAL and SNA. These results could indicate that the SA-MIPs either have a preference for targeting 2,3-SA or the complexity of the glycan chains vary in terms of branching and/or elongations, which could affect the accessibility of the SA-MIPs.

Moreover, to better understand whether the immune cells will discover the SA-MIPs, the particles have been evaluated for uptake by phagocytosing immune cells [116]. The SA-MIPs show binding to murine macrophages, RAW264.7 cells, and by studying uptake by microscopy, it is revealed that the fluorescent SA-MIPs are present inside the cells by the change in color from green fluorescence to orange due to interference with the red phalloidin (Figure 5B).

Interestingly, Medina Rangel et al. used MIPs as chemical antibodies in inhibiting cell-cell adhesion [117]. The MIPs were more potent than commercially available therapeutic antibodies for inhibiting invasion of cells, cell-cell adhesion in aggregation assays and disrupting 3D tumor spheroids as well as inhibiting invasion of HeLa cells. 


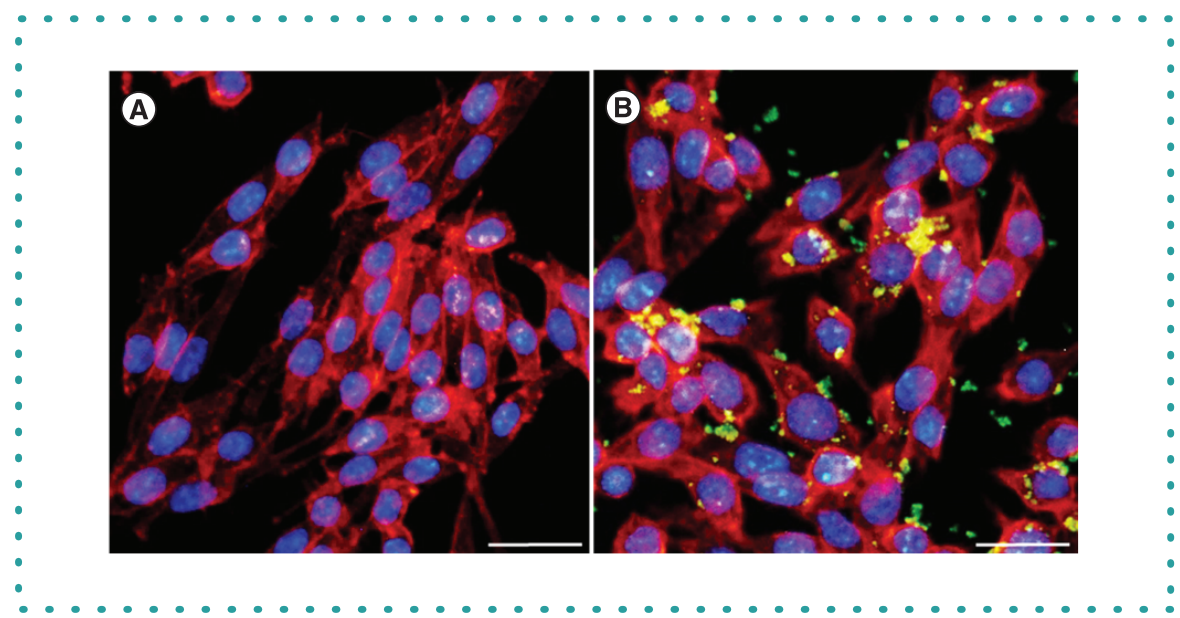

Figure 5. Murine macrophage RAW264.7 cells bind and thereafter ingest fluorescent dSA-molecularly imprinted polymers in a concentration of $0.04 \mathrm{mg} / \mathrm{ml}$ as imaged by fluorescence microscopy. (A) Control RAW264.7 cells. (B) RAW264.7 cells incubated with green fluorescent sialic acid molecularly imprinted polymers. The cytoskeleton is stained in red with phalloidin and the nuclei in blue with DAPI in both images. Scale bar at $10 \mu \mathrm{m}$.

Prostate cancer is the second most common cancer in men worldwide. PSA is the most widely used biomarker for screening prostate cancer, although not without debate [118]. However, PSA is an approved cancer biomarker from the US FDA and is currently used in screening and diagnosis of prostate cancer [119]. Interestingly, several research groups have reported different approaches to detect PSA with MIP technology [120-125]. A nanobiosensor using a MIP on a gold screen-printed electrode in the presence of PSA as a template was fabricated, showing high affinity and specificity for PSA [125].

Zhou et al. used CEA as a target to imprint MIPs [126]. CEA is a glycoprotein serving as a biomarker for colorectal, breast, ovarian, lung, gastric, bladder and pancreatic cancer [127,128]. CEA is produced during fetal development and therefore present in low levels in healthy persons [129]. Orthogonal dual MIP-based plasmonic immunosandwich assay was used to measure the CEA levels in human serum for healthy and colon cancer patients. This method could distinguish between samples from colon cancer patients and healthy individuals [126]. Xing et al. used a similar method to detect neuron-specific enolase in human serum, which is used as a protein biomarker of small cell lung cancer. Dual MIP-based plasmonic immunosandwich assay is a method that could show a specific and sensitive detection of NSE for healthy individuals compared with small cell lung cancer patients. It was concluded that the dual MIP-based plasmonic immunosandwich assay method had several significant advantages compared with commercially available ELISA kits, such as shorter procedures, less time-consuming, lower sample volume requirement and a wider linear range [130].

A surface molecularly imprinted polymer (SMIP) technology recently was integrated with surface-enhanced Raman spectroscopy (SERS) to develop a more powerful quantitative detection strategy (SMIP-SERS). Lin et al. detected CEA levels in human serum for healthy individuals and colon cancer patients with comparison with ELISA. The detection was lower compared with ELISA [102]. The SMIPbased SERS immunoassay was also used for determination of cancer biomarkers such as $\alpha$-fetoprotein, erythropoietin, alkaline, survivin and SA, indicating that the SMIP-SERS analysis provided a promising potential tool for quantitative detection of cancer biomarkers in complex samples $[102,131,132]$. Lin et al. used SERS to detect low concentrations of nasopharyngeal cancer-related DNA, and the results demonstrated that SERS technology could provide a reliable and ultrasensitive method for cancer biomarker detection [133]. Moreover, the MIP technology has been used to measure the levels of drugs in human plasma.

A molecularly imprinted magnetic solid-phase extraction technique using $\mathrm{Fe}_{3} \mathrm{O}_{4} @ \mathrm{MIP}$ nanoparticles was developed and coupled with the LC-MS/MS protocol for simultaneous quantification of the antileukemic agent 6-mercaptopurine and its active metabolite thioguanine in human plasma. The adsorption experiments indicated that MIPs have good recognition properties for 6-mercaptopurine and thioguanine [134]. Obtained data on the levels of these drugs in plasma or serum can give valuable guidelines to more effective therapy [135].

Electrochemical DNA biosensing using MIPs to capture homogenously hybridized DNA nanocomposites was recently evaluated with the determination of brca1 in human serum samples. The results showed excellent selectivity, reproducibility, stability and feasibility in serum analysis of breast cancer patients [136].

\section{In vivo applications of MIPS}

Despite the broad use of MIPs in in vitro applications, the in vivo applications remain challenging because long-term toxicity, biodegradability, biocompatibility and distributions in fluids are not very well known yet.

MIPs are small, stable particles with well-defined characteristics such as size, specificity and fluorescence, and thus they are considered for therapeutic applications. Due to the imprinting procedure, the MIPs are versatile and can be imprinted against various targets 
in vivo such as proteins, glycans or any other moieties present in living organisms. MIP targeting in drug delivery is rapidly increasing, and in this review we have dedicated a separate section for drug delivery systems (DDS) using MIPs.

Hoshino et al. used MIPs for the recognition and neutralization of melittin, a cytolytic peptide that is a principal component of bee venom. The MIPs significantly cleared melittin from the bloodstream of mice and neutralized its activity, which enhanced the survival rate of the mice [137]. A study using MIP nanofibers showed that it is less invasive to living mice compared with in vitro sampling, and that the MIP nanofibers have higher selectivity and capture efficiency, reducing interference of the other compounds in rat liver [138]. Another study has used MIPs as a part of a biosensor to detect the bacterial factor RoxP on skin. Despite the complexity of the environment (e.g., presence of skin proteins), the detection was suitable due to the sensitivity and selectivity of the MIPs [139]. Some MIP applications aim to immobilize the nanoparticles into a layer to detect small organic molecules. Diliën et al. made a MIP layer as a receptor for molecules such as dopamine, cortisol or serotonin and were able to efficiently detect those molecules. The authors suggest integrating this biocompatible setup in a catheter, for example, to provide a convenient method for real-time detection for patients [140].

With the current rapid development of fluorescence imaging technologies coupled with other multimodal approaches (fluorescence/magnetic resonance imaging) [141], MIPs play an important role as a new labeling agent. MIP imaging uses imprinted polymer particles linked with dyes (QDs, carbon dots or conjugated polymers) to yield higher stability or avoid photobleaching, as these issues are rather present when working with fluorescently labeled antibodies [68,142]. Cecchini et al. used MIPs coupled with Cd QDs (QD-MIPs) to localize VEGF, responsible for tumor angiogenesis, in zebrafish. QD-MIPs showed negligible toxic effects and specifically localized proximity of the tumor mass in contrast to nonimprinted QDs [143]. In a different study, MIPs were used as cargo for the anticancer drug doxorubicin and with an embedded photosensitizer producing free radicals after 655-nm laser irradiation to synergistically kill the cancer cells. There were no reported toxic effects of MIPs in mice, and the authors showed significant inhibition of tumor growth after laser irradiation compared with other experimental groups [144].

Dong et al. used glycan-imprinted MIPs as a treatment for breast cancer [145]. In their study, a mouse model was used and showed that MIPs inhibited cell proliferation resulting in a decrease of the tumor volume. The MIPs specifically bind the glycans of HER2, blocking the HER2-dependent signaling pathways via suppressing the dimerization of HER2 that leads to inhibition of breast cancer cell proliferation. The biological toxicity was reported, as well as minimal uptake of the particles by macrophages, suggesting that the MIPs could be used as a novel effective cancer therapy [145]. This application could apply to other cancer therapies, as MIPs can be imprinted for various signaling proteins and glycans in other pathways.

As cancer recurrence contributes to higher mortality, fluorescent nanoparticles were used for punctual detection of relapsed cancer tissue. The nanoparticles were derived from HA, as it is a naturally occurring biopolymer to provide higher biocompatibility and targeting, and ensuring specific uptake. These parameters resulted in higher contrast in tumor tissue compared with healthy surrounding tissue. Additionally, the fluorescence signal was also enhanced and detectable alongside MRI. Since multimodal imaging methods combining MRI and fluorescence imaging are widely used, these nanoparticles would have a great clinical impact [146].

The crucial step for the applications in living systems is the low toxicity of the nanoparticles. The element of the core constituting a nanoparticle determines properties important for the toxicity, as particle surface charge, its size and shape affect the cellular uptake and how the particles interact with organelles and biomolecules $[147,148]$. In addition, the toxicity of the nanoparticles differs between in vitro and in vivo applications. Canfarotta et al. described no toxicity of fluorescent MIPs in different concentrations to human keratinocytes HaCaT and human fibrosarcoma HT1080 cells [149]. Other studies reported low cytotoxic effects of MIPs in vitro after 72-h exposition to breast cancer cell lines MCF7, MDA-MB-231 and mouse fibroblast cell line NIH-3T3, respectively [111,150]. Other studies report low toxicity to different model organisms such as mice or zebrafish $[138,143]$.

To summarize, applications of MIPs in living organisms have limitless possibilities from clearance or blocking of targeted compounds to the possible detection of specific molecules. The enormous potential of MIPs represents their utilization in imaging, as they can be highly specific and easily linked with stains and other probes.

\section{MIIPs \& drug delivery}

DDS need to be able to regulate the amount of drug and the rate at which a drug is released. Ideally, to avoid causing harm to healthy tissues and to increase bioavailability, the drug is transported to the appropriate site into the body before it is released [151]. MIPs have been investigated as drug delivery agents in biological applications. Their high chemical and physical stability, easy and cheap preparation, and long shelf life are just some of the advantages that make them suitable to be used for drug delivery (Figure 6).

The applicability of MIPs as DDS can mostly be accredited to the fact that they can be functionalized to selectively bind to almost any kind of drug molecule with high affinity, resulting in a much higher drug loading capacity [6,152]. MIPs are resistant to extreme pH, temperature and pressure, and they can protect a drug from being degraded by enzymes before they reach their target site [153,154]. Paul et al. synthesized MIPs that were imprinted with insulin as a template molecule to investigate the MIPs' capability of delivering the drug to gastrointestinal cells. Indeed, after being administered in vivo to rats, these MIPs proved to be efficient as oral DDS for diabetic rats. The MIPs protected the drug from degradation, had a better absorption into gastrointestinal epithelial cells and allowed for an extended release of the drug $[155,156]$.

Various concepts have been discussed, investigating the selective binding properties as well as drug loading capacity and release profiles of different MIPs both in vitro and in vivo. The potential of MIPs to prolong the residence time of a drug and to control its 


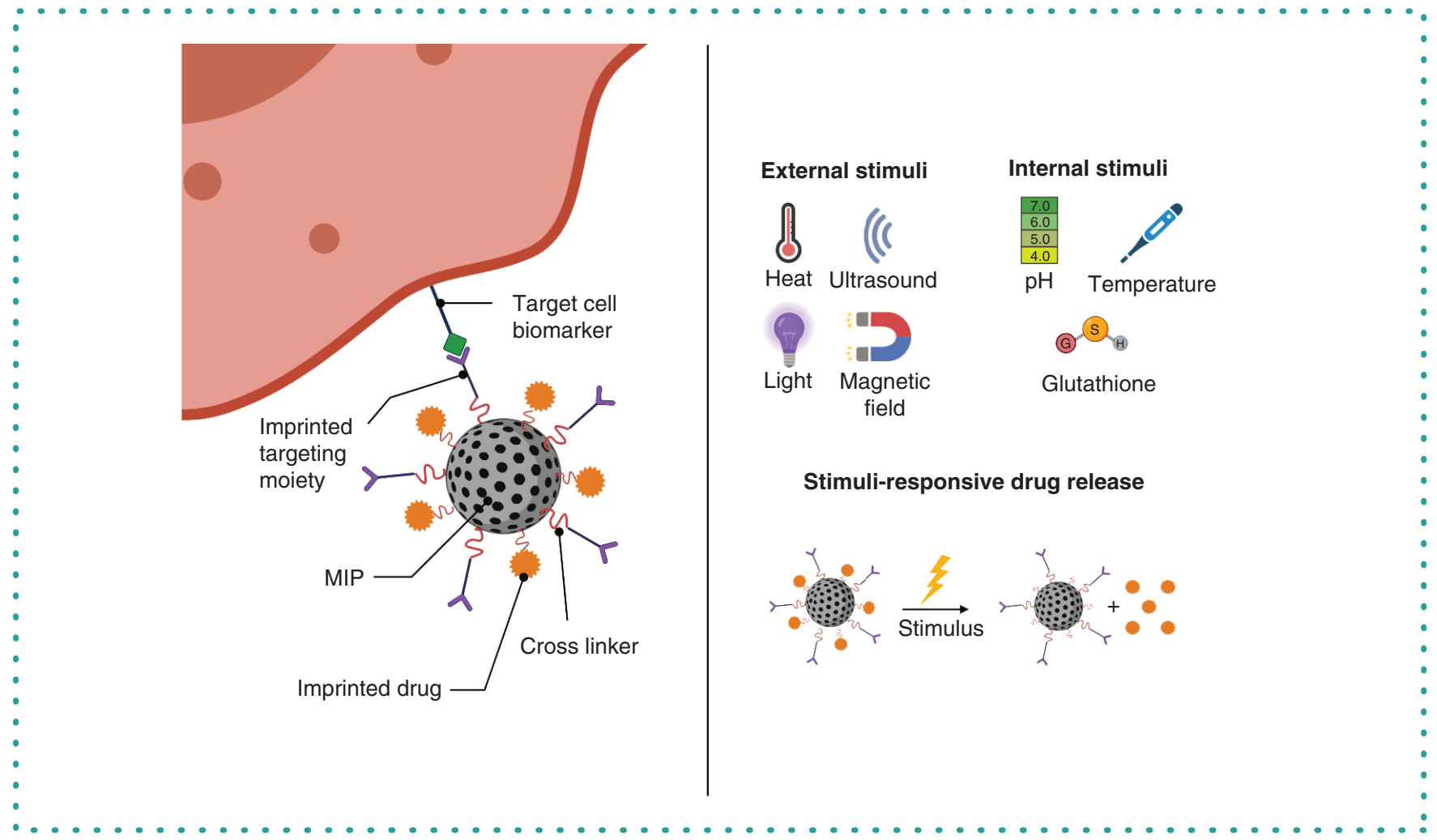

Figure 6. Schematic description of molecularly imprinted polymer-mediated drug delivery. The left image shows a MIP imprinted with a targeting moiety for the cellular biomarker and with the imprinted drug for delivery. The right image shows different external and internal stimuli (upper part) for induction of stimuli-responsive MIP-mediated drug release (lower part).

Created using BioRender.com.

MIP: Molecularly imprinted polymer.

pharmacological activity in the body is one of their best advantages. The sustained release of a drug can reduce the administration frequency and unwanted side effects that might arise due to high concentrations of drug. The most commonly applied method for MIP synthesis in DDS is with the noncovalent approach for interactions between the drug and the functional monomers, as almost any kind of template can be used [153]. The drug release can be sustained by adjusting the strength and kind of interactions between the template and the functional monomers, but even the choice of cross-linker affects the binding and release properties of the MIPs [152,157,158].

MIPs in DDS are being developed for different routes of administration, including oral, intravenous, ocular and transdermal applications [159]. Molecularly imprinted hydrogels or therapeutic contact lenses have been proven to be a good option for sustained ocular drug delivery as an alternative for eye drops that have to be administered several times a day [160,161]. Contact lenses imprinted with the antihistamine ketotifen in rabbits demonstrated a higher bioavailability of the drug, as well as a highly increased residence time of the drug compared with drug-soaked lenses and eye drop treatment [162]. MIPs imprinted with the antibiotic agent vancomycin, for surface coating of implants for sustained drug delivery to prevent bacterial infections, were able to release the drug at a slower rate and for a much longer time course compared with nonimprinted particles [163]. To establish a floating property of the MIP for sustained drug delivery in the GI tract, a liquid crystalline monomer was incorporated into the MIP structure [164,165]. This resulted in a better bioavailability of the drug in vivo in mice and rats due to the longer residence time of the drug in the stomach.

Due to their specificity, MIPs can even bind and release single enantiomers in racemic drugs, which is useful in case one enantiomer is more effective than its opposite. This has been shown by Suedee et al., who investigated the use of enantio-selective MIPs in both oral and transdermal DDS [166-169]. Moreover, molecularly imprinted cellulose membranes were successfully employed for the enantioselective transdermal drug delivery of the ß-blocker S-propranolol through excised rat skin $[169,170]$.

By including responsive copolymers in the MIP synthesis, a drug can be released in response to either internal (e.g., $\mathrm{pH}$ levels or temperature changes) or external (e.g., a near-infrared [NIR] light or a magnetic field) stimuli in the environment [6]. The prodrug sulfasalazine was used to imprint MIPs that were shown to be released by a rapid change from acidic to basic $\mathrm{pH}$, implying their possible use as DDS for sulfasalazine treatment in colon diseases. Even for cancer therapy, MIPs have been investigated as possible drug delivery agents. The more acidic $\mathrm{pH}$ inside the cancer cells makes them a perfect target for $\mathrm{pH}$-induced drug release [171]. Interestingly, nanoparticles imprinted with the anticancer drug paclitaxel showed a higher release of the drug at $\mathrm{pH} 5$ than at $\mathrm{pH}$, making them suitable as a possi- 
ble DDS for paclitaxel [172]. Liu et al. utilized an NIR light-responsive MIP that released paracetamol through porcine skin, controlled by the external stimulus of NIR light, which in the future could lead to the development of a DDS to deep tissues [173].

For targeted drug release, the surfaces of MIPs can be coated with specific ligands that can bind to receptors on target cells and release the drug at a specific site of action $[6,10]$. This reduces side effects and impact on healthy cells, and increases the efficacy of the drug in target cells. Esfandyari-Manesh et al. successfully applied this approach by conjugating polyethylene glycerol-folic acid to paclitaxel-imprinted MIPs for targeted drug release [174]. Zhang et al. investigated the possibility of using p32-epitope-imprinted MIPs loaded with the photosensitizer methylene blue for active targeting and treatment of p32-positive breast tumor cells in mice. The tumor cells had a higher uptake of the MIPs and a higher intracellular concentration of the photosensitizer. When photodynamic therapy (PDT) was applied and the photosensitizer was exposed to light, it produced free radicals that were able to kill the cancer cells and inhibit tumor growth [175]. A combination of both a HER2 epitope, which is overexpressed in breast cancer cells, and the anticancer drug doxorubicin for targeted drug delivery was used to imprint silica nanoparticles, resulting in a higher uptake of MIPs in breast cancer cells compared with nonimprinted particles [176]. Peng et al. imprinted a transmembrane helical peptide onto drug-loaded nanoparticles for pancreatic tumor-targeted PDT in mice. After intravenous administration, the MIPs were able to recognize the transmembrane domain of the target protein in the cancer cell membranes and the drug was released in the cells upon external PDT with a laser [177]. A similar concept was applied by imprinting MIPs with lipopolysaccharides from Pseudomonas aeruginosa and loading them with a photosensitizer for PDT. The results indicated that the MIPs could be used for targeted drug delivery, as the treatment could inhibit bacterial growth [178]. A drawback of PDT is the challenge of delivering light to deep tissues. For this reason, a core-shell MIP with a titanium oxide core that can absorb x-rays and emit visible light during radiotherapy was developed, which activates the photosensitizer imprinted onto the MIP shell to produce free radicals and singlet oxygens to kill the tumor cells [179].

MIPs were imprinted both with the anticancer drug bleomycin and a peptide of the human fibroblast growth factor-inducible 14 that is overexpressed in pancreatic cancer cells. Fibroblast growth factor-inducible 14 on tumor cells was successfully targeted, and the drug was released in response to the acidic $\mathrm{pH}$ inside the cancer cells [159]. Canfarotta et al. used epitope-imprinted MIPs loaded with the anticancer agent doxorubicin to target EGFR, which is overexpressed on cancer cells [180]. A targeted drug delivery and cell death that only occurred in cancer cells could be observed. Double-imprinted nanoparticles selective to SA were synthesized. The MIPs were loaded with a drug sensitive to high concentrations of glutathione (an environment that can be found in cancer cells), to target and treat tumors in vivo in mice [181]. Combining targeting folate-positive cancer cells with loading the MIPs with the glutathione-sensitive drug vinblastine showed that the drug accumulated in tumor tissues while the drug concentration decreased in organs, as compared with the pure drug, decreasing the toxicity of the drug to healthy tissues [182].

Magnetic MIPs have been investigated as controlled drug release agents, as they qualify for the application of an alternative magnetic field that can initiate drug release $[183,184]$. Magnetic doxorubicin-loaded MIPs for controlled stimuli-triggered drug release in cancer cells were investigated. It was demonstrated that upon the application of an alternative magnetic field, the hydrogen bonds between the MIPs and the drug were broken, so the drug was rapidly released in the cells [185]. Hashemi-Moghaddam et al. adopted a similar approach with drug-loaded magnetic MIPs for the controlled release of 5-fluorouracil in a mouse breast cancer model, observing a decrease in tumor growth and increase in the animal life span [186]. The magnetic properties of MIPs can also be used for targeted drug delivery to specific tumors by applying an external magnetic field that directs the MIPs to the tumor site [187,188]. Zavareh et al. investigated the use of doxorubicin-magnetic MIPs for targeted delivery to breast cancer tumors in mice. They implanted magnets subcutaneously at the tumor sites and applied an external magnetic field to direct the MIPs to the site of action, where doxorubicin was released in a sustained manner. Most interestingly, a significant reduction in tumor size and higher survival rates of mice treated with MIPs under a magnetic field could be observed [189].

To summarize, MIPs are promising for use as DDS because they can bind to their template selectively and load high amounts of drug. They can sustain the release of a drug, and the release can be triggered by different stimuli in the environment. MIPs can be directed to different sites in the body for targeted drug release, and the possibilities to apply MIPs as drug delivery agents are numerous, as almost any kind of molecule can be imprinted on the polymer matrix. However, to be able to use MIPs in biological applications in vivo, the MIPs have to be biodegradable and nontoxic [6]. As many of the cross-linkers and functional monomers are toxic, the choice of these becomes limited. Even the choice of solvent for MIP synthesis is important. Many of the MIPs originally synthesized for analytical applications have used organic solvents, as these promote a stronger binding between the functional monomers and template. However, to be able to be used in biological applications, the solvents should be aqueous to mimic biological fluids [190]. The residues of the organic solvents in the MIPs can be toxic, and many drugs and biological compounds are not compatible with organic media [174]. Unfortunately, due to nonspecific hydrophobic interactions between the MIPs and the template, the selectivity decreases in aqueous environments. This problem can be solved by applying hydrophilic monomers in the polymerization process that decrease the interference of water in the binding process between the template and monomers [191,192]. To improve the biodegradability of the MIPs, a biodegradable cross-linker or monomer can be incorporated into the synthesis [6]. MIPs are still under development, and apart from the challenges mentioned, which obviously need to be addressed, MIPs seem to have a promising future in application as DDS. 


\section{Conclusion \& future perspective}

MIP synthesis was developed almost 50 years ago. Initially, the imprinting methods were different, and cellular applications were not possible. Recent work has shown progress with imprinting approaches that give hope for the future. The main advantages of using MIPs are their high stability, including resistance to pressure, high temperatures, extreme $\mathrm{pH}$ and possibility for long-term storage. Moreover, functionalization with fluorophores and short production time are other benefits. MIP synthesis also relies on the template of choice, which can be any biological structure, small enough to create a specific and selective imprint used for a variety of applications. An encouraging development for recognition of cells and molecules within the biomedical area has been witnessed. Interesting approaches are those describing imprinting of whole cells, cell membranes and microorganisms. If successful, this could expand the usability of MIPs for clinical diagnostics and personalized medicine, and in applications of specific diseases, such as cancer.

MIPs have been suggested to replace antibodies and have therefore been named 'molecularly imprinted synthetic antibodies' and 'plastic antibodies'. The use of MIPs as plastic antibodies is particularly important when targeting structures of low immunogenicity, as natural antibodies do not perform well under those circumstances. Areas for further improvement that can be foreseen in MIP development are reduction of nonspecific binding for higher selectivity of the target and reproducible high-quality large-scale production. Indeed, MIPs with high specificity and affinity are desirable in biomedical applications for cellular targets that are lacking promising detection tools. The desired MIPs for future applications need to be specific for the biomarker, reproducible, small and equipped with fluorophores that are functionalized for both in vitro and in vivo applications. Another area of future interest is MIPs with dual functions, such as cellular targeting combined with drug delivery or targeting combined with altered cellular function.

\section{Financial \& competing interests disclosure}

This research was funded by the Swedish Knowledge Foundation (grant no. 20160165), the European Union's Horizon 2020 research and innovation program under the Marie Sklodowska-Curie grant agreement (grant no. 721297), the Biofilms Research Center for Biointerfaces and Malmö University. The authors have no other relevant affiliations or financial involvement with any organization or entity with a financial interest in or financial conflict with the subject matter or materials discussed in the manuscript apart from those disclosed.

No writing assistance was utilized in the production of this manuscript.

\section{Acknowlegdments}

The authors would like to thank Börje Sellergren, Department of Biomedical science, Malmö University, Malmö, Sweden; Sudhirkumar Shinde, School of Chemistry and Chemical Engineering, Queen's University, Belfast, UK; Knut Rurack, Kornelia Gawlitza and Martha Kimani-Wamaitha, Federal Institute for Materials Research and Testing (BAM), Berlin, Germany; and Bo Holmqvist and Anders Brinte, ImaGene-iTAB, Lund, Sweden.

\section{Open access}

This work is licensed under the Creative Commons Attribution 4.0 License. To view a copy of this license, visit http://creativecommons. org/licenses/by/4.0/

\section{References}

1. Mayeux R. Biomarkers: potential uses and limitations. NeuroRx 1(2), 182-188 (2004).

2. Mishra A, Behura A, Mawatwal S et al. Structure-function and application of plant lectins in disease biology and immunity. Food Chem. Toxicol. 134, 110827 (2019).

3. Wulff G, Sarhan A. The use of polymers with enzyme-analogous structures for the resolution of racemates. Angew. Chem. Int. Ed. 11, 341-344 (1972).

4. Zhang Y, Llapashtica K, Shinde S, Sellergren B, El-Schich Z, Gjörloff Wingren A. Determination of cytokine regulated glycan expression by using molecularly imprinted polymers targeting sialic acid. J. Cancer Metastasis Treat. 5, 56 (2019).

5. Refaat D, Aggour MG, Farghali AA et al. Strategies for molecular imprinting and the evolution of MIP nanoparticles as plastic antibodies - synthesis and applications. Int. J. Mol. Sci. 20(24), 6304 (2019).

6. Zhang H. Molecularly imprinted nanoparticles for biomedical applications. Adv. Mater. 32(3), e1806328 (2020).

7. Komiyama M, Mori T, Ariga K. Molecular imprinting: materials nanoarchitectonics with molecular information. Bull. Chem. Soc. Jpn 91(7), 1075-1111 (2018).

8. Vaneckova T, Bezdekova J, Han G, Adam V, Vaculovicova M. Application of molecularly imprinted polymers as artificial receptors for imaging. Acta Biomater. 101, 444-458 (2020).

9. Pan J, Chen W, Ma Y, Pan G. Molecularly imprinted polymers as receptor mimics for selective cell recognition. Chem. Soc. Rev. 47(15), 5574-5587 (2018).

10. Piletsky S, Canfarotta F, Poma A, Bossi AM, Piletsky S. Molecularly imprinted polymers for cell recognition. Trends Biotechnol. 38(4), 368-387 (2020).

11. Cui F, Zhou Z, Zhou HS. Molecularly imprinted polymers and surface imprinted polymers based electrochemical biosensor for infectious diseases. Sensors (Basel) 20(4), 996 (2020).

12. Ahmad OS, Bedwell TS, Esen C, Garcia-Cruz A, Piletsky SA. Molecularly imprinted polymers in electrochemical and optical sensors. Trends Biotechnol. 37(3), 294-309 (2019).

13. Xu J, Miao H, Wang J, Pan G. Molecularly imprinted synthetic antibodies: from chemical design to biomedical applications. Small 16(27), e1906644 (2020).

14. Chen L, Xu S, Li J. Recent advances in molecular imprinting technology: current status, challenges and highlighted applications. Chem. Soc. Rev. 40(5), 2922-2942 (2011).

15. Bruggemann O, Haupt K, Ye L, Yilmaz E, Mosbach K. New configurations and applications of molecularly imprinted polymers. J. Chromatogr. A 889(1-2), 15-24 (2000).

16. Chen L, Wang X, Lu W, Wu X, Li J. Molecular imprinting: perspectives and applications. Chem. Soc. Rev. 45(8), $2137-2211$ (2016).

17. Ji W, Zhang M, Gao Q, Cui L, Chen L, Wang X. Preparation of hydrophilic molecularly imprinted polymers via bulk polymerization combined with hydrolysis of ester groups for selective recognition of iridoid glycosides. Anal. Bioanal. Chem. 408(19), 5319-5328 (2016).

18. Garcia Mayor MA, Paniagua Gonzalez G, Garcinuno Martinez RM, Fernandez Hernando P, Durand Alegria JS. Synthesis and characterization of a molecularly imprinted polymer for the determination of spiramycin in sheep milk. Food Chem. 221, 721-728 (2017).

19. Hua MZ, Feng S, Wang S, Lu X. Rapid detection and quantification of 2,4-dichlorophenoxyacetic acid in milk using molecularly imprinted polymers-surface-enhanced Raman spectroscopy. Food Chem. 258, 254-259 (2018).

20. Samah NA, Sanchez-Martin MJ, Sebastian RM, Valiente M, Lopez-Mesas M. Molecularly imprinted polymer for the removal of diclofenac from water: synthesis and characterization. Sci. Total Environ. 631, 1534-1543 (2018). 
21. Diaz-Bao M, Regal P, Barreiro R, Fente CA, Cepeda A. A facile method for the fabrication of magnetic molecularly imprinted stir-bars: a practical example with aflatoxins in baby foods. J. Chromatogr. A 1471, 51-59 (2016)

22. Lin Z-Z, Zhang H-Y, Li L, Huang Z-Y. Application of magnetic molecularly imprinted polymers in the detection of malachite green in fish samples. React. Funct. Polym. 98, 24-30 (2016).

23. Liu X, Wu F, Au C, Tao Q, Pi M, Zhang W. Synthesis of molecularly imprinted polymer by suspension polymerization for selective extraction of p-hydroxybenzoic acid from water. J. Appl. Polym. Sci. 136(3), 46984 (2019).

24. Alizadeh T, Ganjali MR, Rafiei F, Akhoundian M. Synthesis of nano-sized timolol-imprinted polymer via ultrasonication assisted suspension polymerization in silicon oil and its use for the fabrication of timolol voltammetric sensor. Mater. Sci. Eng. C Mater. Biol. Appl. 77, 300-307 (2017).

25. Song L, He J, Chen N, Huang Z. Combined biocompatible medium with molecularly imprinted polymers for determination of aflatoxins B1 in real sample. J. Sep. Sci. 42(24), 3679-3687 (2019).

26. Zhang Z, Zhang X, Niu D, Li Y, Shi J. Highly efficient and selective removal of trace lead from aqueous solutions by hollow mesoporous silica loaded with molecularly imprinted polymers. J. Hazard Mater. 328, 160-169 (2017).

27. Sun Y, Zhang Y, Ju Z, Niu L, Gong Z, Xu Z. Molecularly imprinted polymers fabricated by Pickering emulsion polymerization for the selective adsorption and separation of quercetin from Spina Gleditsiae. New J. Chem. 43(37), 14747-14755 (2019).

28. Li L, Lin Z-Z, Peng A-H, Zhong H-P, Chen X-M, Huang Z-Y. Biomimetic ELISA detection of malachite green based on magnetic molecularly imprinted polymers. J. Chromatogr. B Analyt. Technol. Biomed. Life Sci. 1035, 25-30 (2016).

29. Zhao X, Cui Y, Wang J, Wang J. Preparation of fluorescent molecularly imprinted polymers via Pickering emulsion interfaces and the application for visual sensing analysis of Listeria monocytogenes. Polymers (Basel) 11(6), 984 (2019).

30. Zhou T, Zhao Q, Zhao L et al. Molecularly imprinted polymers combined with membrane-protected solid-phase extraction to detect triazines in tea samples. Anal. Bioanal. Chem. 410, 5173-5181 (2018)

31. Zhu F, Li L, Xing J. Selective adsorption behavior of $\mathrm{Cd}(\mathrm{II})$ ion imprinted polymers synthesized by microwave-assisted inverse emulsion polymerization: adsorption performance and mechanism. J. Hazard Mater. 321, 103-110 (2017).

32. Zheng X, Xu T, Shi R et al. Preparation of hollow porous molecularly imprinted polymers for N-nitrosamine adsorption. Mater. Lett. 211, 21-23 (2018).

33. Wang Z, Qiu T, Guo L, Ye J, He L, Li X. The building of molecularly imprinted single hole hollow particles: a miniemulsion polymerization approach. Chem. Eng. J. 357, 348-357 (2019).

34. Haginaka J, Nishimura K, Kimachi T, Inamoto K, Takemoto Y, Kobayashi Y. Retention and molecular-recognition mechanisms of molecularly imprinted polymers for promazine derivatives. Talanta 205, 120149 (2019).

35. Wang X, Huang P, Ma X, Du X, Lu X. Magnetic mesoporous molecularly imprinted polymers based on surface precipitation polymerization for selective enrichment of triclosan and triclocarban. J. Chromatogr. A 1537, 35-42 (2018).

36. Nakamura $Y$, Matsunaga $H$, Haginaka J. Preparation of molecularly imprinted polymers for strychnine by precipitation polymerization and multistep swelling and polymerization and their application for the selective extraction of strychnine from nux-vomica extract powder. J. Sep. Sci. 39(8), 1542-1550 (2016).

37. Miura $\mathrm{C}$, Matsunaga $\mathrm{H}$, Haginaka J. Molecularly imprinted polymer for caffeic acid by precipitation polymerization and its application to extraction of caffeic acid and chlorogenic acid from Eucommia ulmodies leaves. J. Pharm. Biomed. Anal. 127, 32-38 (2016).

38. Lu Y, Zhu Y, Zhang Y, Wang K. Synthesizing vitamin E molecularly imprinted polymers via precipitation polymerization. J. Chem. Eng. Data 64(3), 1045-1050 (2019).

39. Phungpanya C, Chaipuang A, Machan T, Watla-Lad K, Thongpoon C, Suwantong 0 . Synthesis of prednisolone molecularly imprinted polymer nanoparticles by precipitation polymerization. Polym. Advan. Technol. 29(12), 3075-3084 (2018)

40. Chen F, Wang J, Chen H, Lu R, Xie X. Microwave-assisted RAFT polymerization of well-constructed magnetic surface molecularly imprinted polymers for specific recognition of benzimidazole residues. Appl. Surf. Sci. 435, 247-255 (2018).

41. Ma Y, Gao J, Zheng $\mathrm{C}$, Zhang $\mathrm{H}$. Well-defined biological sample-compatible molecularly imprinted polymer microspheres by combining RAFT polymerization and thiol-epoxy coupling chemistry. J. Mater. Chem. B 7(15), 2474-2483 (2019).

42. Azizi A, Shahhoseini F, Bottaro CS. Magnetic molecularly imprinted polymers prepared by reversible addition fragmentation chain transfer polymerization for dispersive solid phase extraction of polycyclic aromatic hydrocarbons in water. J. Chromatogr. A 1610, 460534 (2020).

43. Li J, Zhao L, Wei C et al. Preparation of restricted access media molecularly imprinted polymers for efficient separation and enrichment ofloxacin in bovine serum samples. J. Sep. Sci. 42(15), 2491-2499 (2019).

44. Qiu $X$, Xu X-Y, Liang $Y$, Hua $Y$, Guo H. Fabrication of a molecularly imprinted polymer immobilized membrane with nanopores and its application in determination of $\beta 2$-agonists in pork samples. J. Chromatogr. A 1429, 79-85 (2016).

45. Bitar M, Lafarge C, Sok N, Cayot P, Bou-Maroun E. Molecularly imprinted sol-gel polymers for the analysis of iprodione fungicide in wine: synthesis in green solvent. Food Chem. 293, 226-232 (2019).

46. Da Costa Silva RG, Augusto F. Sol-gel molecular imprinted ormosil for solid-phase extraction of methylxanthines. J. Chromatogr. A 1114(2), 216-223 (2006).

47. Ozcan AA, Say R, Denizli A, Ersoz A. L-histidine imprinted synthetic receptor for biochromatography applications. Anal. Chem. 78(20), 7253-7258 (2006).

48. Hantash $\mathrm{J}$, Bartlett $\mathrm{A}$, Oldfield $\mathrm{P}$ et al. Use of an on-line imprinted polymer pre-column, for the liquid chromatographic-UV absorbance determination of carbaryl and its metabolite in complex matrices. J. Chromatogr. A 1125(1), 104-111 (2006).

49. Kempe H, Kempe M. Development and evaluation of spherical molecularly imprinted polymer beads. Anal. Chem. 78(11), 3659-3666 (2006).

50. Zhang H, Dramou P, He H, Tan S, Pham-Huy C, Pan H. Molecularly imprinted stationary phase prepared by reverse micro-emulsion polymerization for selective recognition of gatifloxacin in aqueous media. J. Chromatogr. Sci. 50(6), 499-508 (2012).

51. Haginaka J, Tabo H, Matsunaga H. Preparation of molecularly imprinted polymers for organophosphates and their application to the recognition of organophosphorus compounds and phosphopeptides. Anal. Chim. Acta 748, 1-8 (2012)

52. Pichon V, Chapuis-Hugon F. Role of molecularly imprinted polymers for selective determination of environmental pollutants-a review. Anal. Chim. Acta 622(1-2), 48-61 (2008).

53. Wang J, Cormack PA, Sherrington DC, Khoshdel E. Monodisperse, molecularly imprinted polymer microspheres prepared by precipitation polymerization for affinity separation applications. Angew. Chem. Int. Ed. Engl. 42(43), 5336-5338 (2003).

54. Yoshimatsu K, Lejeune J, Spivak DA, Ye L. Peptide-imprinted polymer microspheres prepared by precipitation polymerization using a single bi-functional monomer. Analyst 134(4), 719-724 (2009).

55. Zamora O, Paniagua EE, Cacho C, Vera-Avila LE, Perez-Conde C. Determination of benzimidazole fungicides in water samples by on-line MISPE-HPLC. Anal. Bioanal. Chem. 393(6-7), 1745-1753 (2009).

56. Shinde S, El-Schich Z, Malakpour A et al. Sialic acid-imprinted fluorescent core-shell particles for selective labeling of cell surface glycans. J. Am. Chem. Soc. 137(43), 13908-13912 (2015).

57. Boonpangrak S, Whitcombe MJ, Prachayasittikul V, Mosbach K, Ye L. Preparation of molecularly imprinted polymers using nitroxide-mediated living radical polymerization. Biosens. Bioelectron. 22(3), 349-354 (2006).

58. Wang HJ, Zhou WH, Yin XF, Zhuang ZX, Yang HH, Wang XR. Template synthesized molecularly imprinted polymer nanotube membranes for chemical separations. J. Am. Chem. Soc. 128(50), 15954-15955 (2006).

59. Walcarius A, Collinson MM. Analytical chemistry with silica sol-gels: traditional routes to new materials for chemical analysis. Annu. Rev. Anal. Chem. 2, 121-143 (2009).

60. Moein MM, Abdel-Rehim A, Abdel-Rehim M. Recent applications of molecularly imprinted sol-gel methodology in sample preparation. Molecules 24(16), 2889 (2019).

61. Dai S, Shin Y, Barnes C, Toth L. Enhancement of uranyl adsorption capacity and selectivity on silica sol-gel glasses via molecular imprinting. Chem. Mater. 9(11), 2521-2525 (1997).

62. Li Y, Li X, Li Y, Dong C, Jin P, Qi J. Selective recognition of veterinary drugs residues by artificial antibodies designed using a computational approach. Biomaterials 30(18), 3205-3211 (2009).

63. He C, Long Y, Pan J, Li K, Liu F. Molecularly imprinted silica prepared with immiscible ionic liquid as solvent and porogen for selective recognition of testosterone. Talanta 74(5), 1126-1131 (2008).

64. Ansari S, Karimi M. Recent configurations and progressive uses of magnetic molecularly imprinted polymers for drug analysis. Talanta 167, 470-485 (2017).

65. Ming W, Wang X, Lu W et al. Magnetic molecularly imprinted polymers for the fluorescent detection of trace 17 $\beta$-estradiol in environmental water. Sens. Actuator. B Chem. 238 , $1309-1315$ (2017)

66. Diltemiz SE, Say R, Buyuktiryaki S, Hur D, Denizli A, Ersoz A. Quantum dot nanocrystals having guanosine imprinted nanoshell for DNA recognition. Talanta 75(4), 890-896 (2008). 
67. Wang HF, He Y, Ji TR, Yan XP. Surface molecular imprinting on Mn-doped ZnS quantum dots for room-temperature phosphorescence optosensing of pentachlorophenol in water. Anal. Chem. 81(4), 1615-1621 (2009).

68. Ensafi AA, Nasr-Esfahani P, Rezaei B. Synthesis of molecularly imprinted polymer on carbon quantum dots as an optical sensor for selective fluorescent determination of promethazine hydrochloride. Sens. Actuators B Chem. 257, 889-896 (2018).

69. Sun X, Liu Y, Niu N, Chen L. Synthesis of molecularly imprinted fluorescent probe based on biomass-derived carbon quantum dots for detection of mesotrione. Anal. Bioanal. Chem. 411(21), 5519-5530 (2019).

70. Fang G-Z, Tan J, Yan X-P. An ion-imprinted functionalized silica gel sorbent prepared by a surface imprinting technique combined with a sol-gel process for selective solid-phase extraction of cadmium (II). Anal. Chem. 77(6), 1734-1739 (2005).

71. Li F, Li X-M, Zhang S-S. One-pot preparation of silica-supported hybrid immobilized metal affinity adsorbent with macroporous surface based on surface imprinting coating technique combined with polysaccharide incorporated sol-gel process. J. Chromatogr. A 1129(2), 223-230 (2006).

72. Du K, Luo Z, Guo P et al. Preparation and evaluation of a molecularly imprinted sol-gel material as the solid-phase extraction adsorbents for the specific recognition of cloxacilloic acid in cloxacillin. J. Sep. Sci. 39(3), 483-489 (2016).

73. Liao S, Zhang W, Long W, Hou D, Yang X, Tan N. Adsorption characteristics, recognition properties, and preliminary application of nordihydroguaiaretic acid molecularly imprinted polymers prepared by sol-gel surface imprinting technology. Appl. Surf. Sci. 364, 579-588 (2016).

74. Peng M, Xiang H, Hu X, Shi S, Chen X. Boronate affinity-based surface molecularly imprinted polymers using glucose as fragment template for excellent recognition of glucosides. J. Chromatogr. A 1474, 8-13 (2016).

75. Silika Z, Dan Isoterma KMK. Glucose sulfate imprinted polymer prepared by sol-gel process on silica microparticles surface: kinetic modeling and isotherm studies. Malaysian J. Anal. Sci. 19(4), 799-807 (2015).

76. Li D, Tu T, Yang M, Xu C. Efficient preparation of surface imprinted magnetic nanoparticles using poly (2-anilinoethanol) as imprinting coating for the selective recognition of glycoprotein. Talanta 184, 316-324 (2018).

77. Li F, Li J, Zhang S. Molecularly imprinted polymer grafted on polysaccharide microsphere surface by the sol-gel process for protein recognition. Talanta 74(5), 1247-1255 (2008).

78. Bie Z, Chen Y, Ye J, Wang S, Liu Z. Boronate-affinity glycan-oriented surface imprinting: a new strategy to mimic lectins for the recognition of an intact glycoprotein and its characteristic fragments. Angew. Chem. Int. Ed. 54(35), 10211-10215 (2015).

79. Cieplak M, Kutner W. Artificial biosensors: how can molecular imprinting mimic biorecognition? Trends Biotechnol. 34(11), 922-941 (2016).

80. Selvolini G, Marrazza G. MIP-based sensors: promising new tools for cancer biomarker determination. Sensors 17(4), 718 (2017).

81. Fu G, Zhao J, Yu H, Liu L, He B. Bovine serum albumin-imprinted polymer gels prepared by graft copolymerization of acrylamide on chitosan. React. Funct. Polym. 65(5), 442-450 (2007).

82. Wang X, Dong J, Ming H, Ai S. Sensing of glycoprotein via a biomimetic sensor based on molecularly imprinted polymers and graphene-Au nanoparticles. Analyst 138(4), 1219-1225 (2013).

83. Kan X, Zhao Q, Shao D, Geng Z, Wang Z, Zhu JJ. Preparation and recognition properties of bovine hemoglobin magnetic molecularly imprinted polymers. J. Phys. Chem. B 114(11), 3999-4004 (2010).

84. Wang Y, Zhang Q, Ren Y, Jing L, Wei T. Molecularly imprinted polymer thin film based surface plasmon resonance sensor to detect hemoglobin. Chem. Res. Chin. Univ. 30(1), 42-48 (2014).

85. Bosserdt M, Erdossy J, Lautner $\mathrm{G}$ et al. Microelectrospotting as a new method for electrosynthesis of surface-imprinted polymer microarrays for protein recognition. Biosens. Bioelectron. 73, 123-129 (2015).

86. Bognár J, Szucs J, Dorkõ Z, Horváth V, Gyurcsányi RE. Nanosphere lithography as a versatile method to generate surface-imprinted polymer films for selective protein recognition. Adv. Funct. Mater. 23(37), 4641 (2013).

87. Gast M, Kühner S, Sobek H, Walther P, Mizaikoff B. Enhanced selectivity by passivation: molecular imprints for viruses with exceptional binding properties. Anal. Chem. 90(9), 5576-5585 (2018).

88. Bai W, Spivak DA. A double-imprinted diffraction-grating sensor based on a virus-responsive super-aptamer hydrogel derived from an impure extract. Angew. Chem. Int. Ed. 53(8), 20952098 (2014).

89. Gast M, Sobek H, Mizaikoff B. Advances in imprinting strategies for selective virus recognition a review. Trends Anal. Chem. 114, 218-232 (2019).

90. Eersels K, Diliën H, Lowdon JW et al. A novel biomimetic tool for assessing vitamin K status based on molecularly imprinted polymers. Nutrients 10(6), 751 (2018).

91. Afsarimanesh N, Mukhopadhyay SC, Kruger M. Molecularly imprinted polymer-based electrochemical biosensor for bone loss detection. IEEE Trans. Biomed. Eng. 65(6), 1264-1271 (2018).

92. Moreira FTC, Sharma S, Dutra RaF, Noronha JPC, Cass AEG, Sales MGF. Protein-responsive polymers for point-of-care detection of cardiac biomarker. Sens. Actuators B Chem. 196, 123-132 (2014).

93. Alizadeh T, Nayeri S. An enzyme-free sensing platform based on molecularly imprinted polymer/MWCNT composite for sub-micromolar-level determination of pyruvic acid as a cancer biomarker. Anal. Bioanal. Chem. 412, 657-667 (2020).

94. El-Schich Z, Abdullah M, Shinde S et al. Different expression levels of glycans on leukemic cells-a novel screening method with molecularly imprinted polymers (MIP) targeting sialic acid. Tumour Biol. 37(10), 13763-13768 (2016).

95. Johari-Ahar M, Karami P, Ghanei M, Afkhami A, Bagheri H. Development of a molecularly imprinted polymer tailored on disposable screen-printed electrodes for dual detection of EGFR and VEGF using nano-liposomal amplification strategy. Biosens. Bioelectron. 107, 26-33 (2018).

96. Rossetti C, Ore OG, Sellergren B, Halvorsen TG, Reubsaet L. Exploring the peptide retention mechanism in molecularly imprinted polymers. Anal. Bioanal. Chem. 409(24), 5631-5643 (2017).

97. Rossetti C, Switnicka-Plak MA, Grønhaug Halvorsen T, Cormack PaG, Sellergren B, Reubsaet L. Automated protein biomarker analysis: on-line extraction of clinical samples by molecularly imprinted polymers. Sci. Rep. 7, 44298 (2017).

98. Rossetti C, Levernæs MCS, Reubsaet L, Halvorsen TG. Evaluation of affinity-based serum clean-up in mass spectrometric analysis: plastic vs monoclonal antibodies. J. Chromatogr. A 1471, 19-26 (2016).

99. Jiang W, Liu L, Chen Y. Simultaneous detection of human C-terminal p53 isoforms by single template molecularly imprinted polymers (MIPs) coupled with liquid chromatography-tandem mass spectrometry (LC-MS/MS)-based targeted proteomics. Anal. Chem. 90(5), 3058-3066 (2018).

100. Ribeiro JA, Pereira CM, Silva AF, Sales MGF. Disposable electrochemical detection of breast cancer tumour marker CA 15-3 using poly(Toluidine Blue) as imprinted polymer receptor Biosens. Bioelectron. 109, 246-254 (2018).

101. Rebelo TSCR, Costa R, Brandão ATSC, Silva AF, Sales MGF, Pereira CM. Molecularly imprinted polymer SPE sensor for analysis of CA-125 on serum. Anal. Chim. Acta 1082, 126-135 (2019).

102. Lin X, Wang Y, Wang L et al. Interference-free and high precision biosensor based on surface enhanced Raman spectroscopy integrated with surface molecularly imprinted polymer technology for tumor biomarker detection in human blood. Biosens. Bioelectron. 143, 111599 (2019).

103. Panagiotopoulou M, Salinas Y, Beyazit S et al. Molecularly imprinted polymer coated quantum dots for multiplexed cell targeting and imaging. Angew. Chem. Int. Ed. Engl. 55(29), 82448248 (2016).

104. Wang S, Yin D, Wang W et al. Targeting and imaging of cancer cells via monosaccharide-imprinted fluorescent nanoparticles. Sci. Rep. 6, 22757 (2016).

105. Kunath S, Marchyk N, Haupt K, Feller K-H. Multi-objective optimization and design of experiments as tools to tailor molecularly imprinted polymers specific for glucuronic acid. Talanta 105, 211-218 (2013).

106. Panagiotopoulou M, Kunath S, Medina-Rangel PX, Haupt K, Bui BTS. Fluorescent molecularly imprinted polymers as plastic antibodies for selective labeling and imaging of hyaluronan and sialic acid on fixed and living cells. Biosens. Bioelectron. 88, 85-93 (2017).

107. Wang S, Wen Y, Wang Y, Ma Y, Liu Z. Pattern recognition of cells via multiplexed imaging with monosaccharide-imprinted quantum dots. Anal. Chem. 89(10), 5646-5652 (2017).

108. Bie Z, Xing R, He X, Ma Y, Chen Y, Liu Z. Precision imprinting of glycopeptides for facile preparation of glycan-specific artificial antibodies. Anal. Chem. 90(16), 9845-9852 (2018).

109. Demir B, Lemberger MM, Panagiotopoulou M et al. Tracking hyaluronan: molecularly imprinted polymer coated carbon dots for cancer cell targeting and imaging. ACS Appl. Mater. Interfaces 10(4), 3305-3313 (2018).

110. Rangel PXM, Laclef S, Xu J et al. Solid-phase synthesis of molecularly imprinted polymer nanolabels: affinity tools for cellular bioimaging of glycans. Sci. Rep. 9(1), 1-9 (2019).

111. Patel M, Feith M, Janicke B, Alm K, El-Schich Z. Evaluation of the impact of imprinted polymer particles on morphology and motility of breast cancer cells by using digital holographic cytometry. Appl. Sci. 10(3), 750 (2020).

112. Saylan Y, Denizli A. molecularly imprinted polymer-based microfluidic systems for point-of-care applications. Micromachines 10(11), 766 (2019). 
113. Golabi M, Kuralay F, Jager EWH, Beni V, Turner APF. Electrochemical bacterial detection using poly(3-aminophenylboronic acid)-based imprinted polymer. Biosens. Bioelectron. 93, 87-93 (2017).

114. Shen X, Svensson Bonde J, Kamra T et al. Bacterial imprinting at pickering emulsion interfaces. Angew. Chem. Int. Ed. Engl. 53(40), 10687-10690 (2014).

115. Peixoto A, Relvas-Santos M, Azevedo R, Santos LL, Ferreira JA. Protein glycosylation and tumor microenvironment alterations driving cancer hallmarks. Front. Oncol. 9, 380 (2019).

116. Sternbæk L, Kimani Wamaitha M, Gawlitza K, Janicke B, Alm K, Wingren Gjörloff A. Digital holographic microscopy: macrophage uptake of nanoprobes. Imaging Microsc. 1, 21-23 (2019).

117. Medina Rangel PX, Moroni E, Merlier F et al. Chemical antibody mimics inhibit cadherin-mediated cell-cell adhesion: a promising strategy for cancer therapy. Angew. Chem. Int. Ed. 59(7), 2816-2822 (2020).

118. Duffy MJ. Biomarkers for prostate cancer: prostate-specific antigen and beyond. Clin. Chem. Lab. Med. 58(3), 326-339 (2020).

119. Linton HJ, Marks LS, Millar LS, Knott CL, Rittenhouse HG, Mikolajczyk SD. Benign prostate-specific antigen (BPSA) in serum is increased in benign prostate disease. Clin. Chem. 49(2), 253-259 (2003).

120. Ertürk G, Özen H, Tümer MA, Mattiasson B, Denizli A. Microcontact imprinting based surface plasmon resonance (SPR) biosensor for real-time and ultrasensitive detection of prostate specific antigen (PSA) from clinical samples. Sens. Actuators B Chem. 224, 823-832 (2016).

121. Ertürk G, Hedström M, Tümer MA, Denizli A, Mattiasson B. Real-time prostate-specific antigen detection with prostate-specific antigen imprinted capacitive biosensors. Anal. Chim. Acta 891, 120-129 (2015).

122. Jolly P, Tamboli V, Harniman RL, Estrela P, Allender CJ, Bowen JL. Aptamer-MIP hybrid receptor for highly sensitive electrochemical detection of prostate specific antigen. Biosens. Bioelectron. 75, 188-195 (2016).

123. Rebelo TSCR, Santos C, Costa-Rodrigues J, Fernandes MH, Noronha JP, Sales MGF. Novel prostate specific antigen plastic antibody designed with charged binding sites for an improved protein binding and its application in a biosensor of potentiometric transduction. Electrochim. Acta 132, 142-150 (2014).

124. Patra S, Roy E, Madhuri R, Sharma PK. Nano-iniferter based imprinted sensor for ultra trace level detection of prostate-specific antigen in both men and women. Biosens. Bioelectron. 66 , 1-10 (2015).

125. Yazdani Z, Yadegari H, Heli H. A molecularly imprinted electrochemical nanobiosensor for prostate specific antigen determination. Anal. Biochem. 566, 116-125 (2019).

126. Zhou L, Wang Y, Xing R et al. Orthogonal dual molecularly imprinted polymer-based plasmonic immunosandwich assay: a double characteristic recognition strategy for specific detection of glycoproteins. Biosens. Bioelectron. 145, 111729 (2019).

127. Han Q, Wang R, Xing B et al. Label-free photoelectrochemical immunoassay for CEA detection based on CdS sensitized W03@ BiOI heterostructure nanocomposite. Biosens. Bioelectron. 99, 493-499 (2018).

128. Konishi T, Shimada Y, Hsu M et al. Association of preoperative and postoperative serum carcinoembryonic antigen and colon cancer outcome. JAMA Oncol. 4(3), 309-315 (2018).

129. Hall C, Clarke L, Pal A et al. A review of the role of carcinoembryonic antigen in clinical practice. Ann. Coloproctol. 35(6), 294-305 (2019).

130. Xing R, Wen Y, Dong Y, Wang Y, Zhang Q, Liu Z. Dual molecularly imprinted polymer-based plasmonic immunosandwich assay for the specific and sensitive detection of protein biomarkers. Anal. Chem. 91(15), 9993-10000 (2019).

131. Tu X, Muhammad P, Liu J et al. Molecularly imprinted polymer-based plasmonic immunosandwich assay for fast and ultrasensitive determination of trace glycoproteins in complex samples. Anal. Chem. 88(24), 12363-12370 (2016).

132. Ye J, Chen Y, Liu Z. A boronate affinity sandwich assay: an appealing alternative to immunoassays for the determination of glycoproteins. Angew. Chem. Int. Ed. 53(39), 10386-10389 (2014).

133. Lin D, Gong T, Qiu S et al. A dual signal amplification nanosensor based on SERS technology for detection of tumor-related DNA. Chem. Commun. (Camb.) 55(11), 1548-1551 (2019).

134. Attallah OA, Al-Ghobashy MA, Ayoub AT, Nebsen M. Magnetic molecularly imprinted polymer nanoparticles for simultaneous extraction and determination of 6-mercaptopurine and its active metabolite thioguanine in human plasma. J. Chromatogr. A 1561, 28-38 (2018).

135. Finkel JM. A fluorometric method for the estimation of 6-mercaptopurine in serum. Anal. Biochem. 21(3), 362-371 (1967).

136. You M, Yang S, Tang W, Zhang F, He P. Molecularly imprinted polymers-based electrochemical DNA biosensor for the determination of BRCA-1 amplified by SiO2@Ag. Biosens. Bioelectron. 112, 72-78 (2018).

137. Hoshino $\mathrm{Y}$, Koide $\mathrm{H}$, Urakami T et al. Recognition, neutralization, and clearance of target peptides in the bloodstream of living mice by molecularly imprinted polymer nanoparticles: a plastic antibody. J. Am. Chem. Soc. 132(19), 6644-6645 (2010).

138. Gao D, Wang D-D, Zhang Q et al. In vivo selective capture and rapid identification of luteolin and its metabolites in rat livers by molecularly imprinted solid-phase microextraction. J. Agric. Food Chem. 65(6), 1158-1166 (2017).

139. Ertürk Bergdahl G, Andersson T, Allhorn M, Yngman S, Timm R, Lood R. In vivo detection and absolute quantification of a secreted bacterial factor from skin using molecularly imprinted polymers in a surface plasmon resonance biosensor for improved diagnostic abilities. ACS Sens. 4(3), 717-725 (2019).

140. Diliën H, Peeters M, Royakkers J et al. Label-free detection of small organic molecules by molecularly imprinted polymer functionalized thermocouples: toward in vivo applications. ACS Sens. 2(4), 583-589 (2017).

141. Key J, Leary JF. Nanoparticles for multimodal in vivo imaging in nanomedicine. Int. J. Nanomed. 9(1), 711-726 (2014).

142. Wang Z, Long R, Peng M, Li T, Shi S. Molecularly imprinted polymers-coated CdTe quantum dots for highly sensitive and selective fluorescent determination of ferulic acid. J. Anal. Methods Chem. 2019, 1505878 (2019).

143. Cecchini A, Raffa V, Canfarotta F et al. In vivo recognition of human vascular endothelial growth factor by molecularly imprinted polymers. Nano Lett. 17(4), 2307-2312 (2017).

144. Peng H, Qin Y-T, He X-W, Li W-Y, Zhang Y-K. Epitope molecularly imprinted polymer nanoparticles for chemo-/photodynamic synergistic cancer therapy guided by targeted fluorescence imaging. ACS Appl. Mater. Interfaces 12(11), 13360-13370 (2020).

145. Dong Y, Li W, Gu Z et al. Inhibition of HER2-positive breast cancer growth by blocking the HER2 signaling pathway with HER2 glycan-imprinted nanoparticles. Angew. Chem. Int. Ed. Engl. 58(31), 10621-10625 (2019).

146. Payne WM, Hill TK, Svechkarev D, Holmes MB, Sajja BR, Mohs AM. Multimodal imaging nanoparticles derived from hyaluronic acid for integrated preoperative and intraoperative cancer imaging. Contrast Media Mol. Imaging 2017, 9616791 (2017).

147. Huang Y-W, Cambre M, Lee H-J. The toxicity of nanoparticles depends on multiple molecular and physicochemical mechanisms. Int. J. Mol. Sci. 18(12), 2702 (2017).

148. Hoshyar N, Gray S, Han H, Bao G. The effect of nanoparticle size on in vivo pharmacokinetics and cellular interaction. Nanomedicine 11(6), 673-692 (2016).

149. Canfarotta F, Waters A, Sadler R et al. Biocompatibility and internalization of molecularly imprinted nanoparticles. Nano Res. 9(11), 3463-3477 (2016).

150. Rechichi A, Cristallini C, Vitale U et al. New biomedical devices with selective peptide recognition properties. Part 1: characterization and cytotoxicity of molecularly imprinted polymers. J. Cell. Mol. Med. 11(6), 1367-1376 (2007).

151. Li C, Wang J, Wang Y et al. Recent progress in drug delivery. Acta Pharm. Sin. B 9(6), 1145-1162 (2019).

152. Tamahkar E, Bakhshpour M, Denizli A. Molecularly imprinted composite bacterial cellulose nanofibers for antibiotic release. J. Biomater. Sci. Polymer Ed. 30(6), 450-461 (2019).

153. Bodoki AE, lacob B-C, Bodoki E. Perspectives of molecularly imprinted polymer-based drug delivery systems in cancer therapy. Polymers (Basel) 11(12), 2085 (2019).

154. Marcelo G, Ferreira IC, Viveiros R, Casimiro T. Development of itaconic acid-based molecular imprinted polymers using supercritical fluid technology for pH-triggered drug delivery. Int. J. Pharm. 542(1), 125-131 (2018).

155. Paul PK, Treetong A, Suedee R. Biomimetic insulin-imprinted polymer nanoparticles as a potential oral drug delivery system. Acta Pharm. 67(2), 149-168 (2017).

156. Paul PK, Nopparat J, Nuanplub M, Treetong A, Suedee R. Improvement in insulin absorption into gastrointestinal epithelial cells by using molecularly imprinted polymer nanoparticles: microscopic evaluation and ultrastructure. Int. J. Pharm. 530(1), 279-290 (2017).

157. Cegłowski M, Kurczewska J, Ruszkowski P, Liberska J, Schroeder G. The influence of cross-linking agent onto adsorption properties, release behavior and cytotoxicity of doxorubicinimprinted microparticles. Colloid. Surf. B Biointerfaces 182, 110379 (2019).

158. Cirillo G, Curcio M, Parisi Ol et al. Gastro-intestinal sustained release of phytic acid by molecularly imprinted microparticles. Pharm. Dev. Tech. 15(5), 526-531 (2010).

159. Jia C, Zhang M, Zhang Y et al. Preparation of dual-template epitope imprinted polymers for targeted fluorescence imaging and targeted drug delivery to pancreatic cancer BxPC-3 cells. ACS Appl. Mater. Interfaces 11(35), 32431-32440 (2019).

160. Omranipour HM, Sajadi Tabassi SA, Kowsari R, Rad MS, Mohajeri SA. Brimonidine imprinted hydrogels and evaluation of their binding and releasing properties as new ocular drug delivery systems. Curr. Drug Deliv. 12(6), 717-725 (2015).

161. Ali M, Byrne ME. Controlled release of high molecular weight hyaluronic acid from molecularly imprinted hydrogel contact lenses. Pharm. Res. 26(3), 714-726 (2009). 
162. Tieppo A, White CJ, Paine AC, Voyles ML, McBride MK, Byrne ME. Sustained in vivo release from imprinted therapeutic contact lenses. J. Control. Release 157(3), 391-397 (2012). 163. Mao C, Xie X, Liu X et al. The controlled drug release by pH-sensitive molecularly imprinted nanospheres for enhanced antibacterial activity. Mat. Sci. Eng. C Mater. 77, 84-91 (2017). 164. Zhang L-P, Tan X-X, Huang Y-P, Liu Z-S. Floating liquid crystalline molecularly imprinted polymer coated carbon nanotubes for levofloxacin delivery. Eur. J. Pharm. Biopharm. 127, 150-158 (2018).

165. Mo CE, Chai MH, Zhang LP, Ran RX, Huang YP, Liu ZS. Floating molecularly imprinted polymers based on liquid crystalline and polyhedral oligomeric silsesquioxanes for capecitabine sustained release. Int. J. Pharm. 557, 293-303 (2019).

166. Suedee R, Srichana T, Martin GP. Evaluation of matrices containing molecularly imprinted polymers in the enantioselective-controlled delivery of beta-blockers. J. Control. Release 66(2-3), 135-147 (2000).

167. Suedee R, Srichana T, Rattananont T. Enantioselective release of controlled delivery granules based on molecularly imprinted polymers. Drug Deliv. 9(1), 19-30 (2002).

168. Suedee R, Bodhibukkana C, Tangthong N, Amnuaikit C, Kaewnopparat S, Srichana T. Development of a reservoir-type transdermal enantioselective-controlled delivery system for racemic propranolol using a molecularly imprinted polymer composite membrane. J. Control. Release 129(3), 170-178 (2008).

169. Suedee R, Jantarat C, Lindner W, Viernstein H, Songkro S, Srichana T. Development of a pH-responsive drug delivery system for enantioselective-controlled delivery of racemic drugs. J. Control. Release 142(1), 122-131 (2010).

170. Jantarat C, Tangthong N, Songkro S, Martin GP, Suedee R. S-propranolol imprinted polymer nanoparticle-on-microsphere composite porous cellulose membrane for the enantioselectively controlled delivery of racemic propranolol. Int. J. Pharm. 349(1), 212-225 (2008).

171. Puoci F, lemma F, Muzzalupo R et al. Spherical molecularly imprinted polymers (SMIPs) via a novel precipitation polymerization in the controlled delivery of sulfasalazine. Macromol. Biosci. 4(1), 22-26 (2004).

172. Bai J, Zhang Y, Chen L et al. Synthesis and characterization of paclitaxel-imprinted microparticles for controlled release of an anticancer drug. Mat. Sci. Eng. C Mater. 92, 338-348 (2018).

173. Liu L-T, Chen M-J, Yang H-L et al. An NIR-light-responsive surface molecularly imprinted polymer for photoregulated drug release in aqueous solution through porcine tissue. Mat. Sci. Eng. C Mater. 106, 110253 (2020)

174. Esfandyari-Manesh M, Javanbakht M, Dinarvand R, Atyabi F. Molecularly imprinted nanoparticles prepared by miniemulsion polymerization as selective receptors and new carriers for the sustained release of carbamazepine. J. Mater. Sci. Mater. Med. 23(4), 963-972 (2012).

175. Zhang Y, Deng C, Liu S et al. Active targeting of tumors through conformational epitope imprinting. Angew. Chem. Int. Ed. Engl. 54(17), 5157-5160 (2015).

176. Wang HY, Cao PP, He ZY et al. Targeted imaging and targeted therapy of breast cancer cells via fluorescent double template-imprinted polymer coated silicon nanoparticles by an epitope approach. Nanoscale 11(36), 17018-17030 (2019).

177. Peng S, Wang Y, Li N, Li C. Enhanced cellular uptake and tumor penetration of nanoparticles by imprinting the "hidden" part of membrane receptors for targeted drug delivery. Chem. Commun. (Camb.) 53(81), 11114-11117 (2017).

178. Long Y, Li Z, Bi Q et al. Novel polymeric nanoparticles targeting the lipopolysaccharides of Pseudomonas aeruginosa. Int. J. Pharm. 502(1), 232-241 (2016).

179. Bakhshizadeh M, Sazgarnia A, Seifi M, Hadizadeh F, Rajabzadeh G, Mohajeri SA. TiO2-based mitoxantrone imprinted poly (methacrylic acid-co-polycaprolctone diacrylate) nanoparticles as a drug delivery system. Curr. Pharm. Des. 23(18), 2685-2694 (2017).

180. Canfarotta F, Lezina L, Guerreiro A et al. Specific drug delivery to cancer cells with double-imprinted nanoparticles against epidermal growth factor receptor. Nano Lett. 18(8), 4641-4646 (2018).

181. Liu T, Qiao Z, Wang J et al. Molecular imprinted S-nitrosothiols nanoparticles for nitric oxide control release as cancer target chemotherapy. Colloid. Surf. B Biointerfaces 173 , 356-365 (2019).

182. Zhu Y, Liu R, Huang H, Zhu Q. Vinblastine-loaded nanoparticles with enhanced tumor-targeting efficiency and decreasing toxicity: developed by one-step molecular imprinting process. Mol. Pharm. 16(6), 2675-2689 (2019).

183. Kan X, Geng Z, Zhao Y, Wang Z, Zhu JJ. Magnetic molecularly imprinted polymer for aspirin recognition and controlled release. Nanotechnology 20(16), 165601 (2009).

184. Cazares-Cortes E, Nerantzaki M, Fresnais J, Wilhelm C, Griffete N, Ménager C. Magnetic nanoparticles create hot spots in polymer matrix for controlled drug release. Nanomaterials (Basel) 8(10), 850 (2018).

185. Griffete N, Fresnais J, Espinosa A, Wilhelm C, Bée A, Ménager C. Design of magnetic molecularly imprinted polymer nanoparticles for controlled release of doxorubicin under an alternative magnetic field in athermal conditions. Nanoscale 7(45), 18891-18896 (2015).

186. Hashemi-Moghaddam H, Kazemi-Bagsangani S, Jamili M, Zavareh S. Evaluation of magnetic nanoparticles coated by 5 -fluorouracil imprinted polymer for controlled drug delivery in mouse breast cancer model. Int. J. Pharm. 497(1), 228-238 (2016).

187. Li L, Chen L, Zhang H, Yang Y, Liu X, Chen Y. Temperature and magnetism bi-responsive molecularly imprinted polymers: preparation, adsorption mechanism and properties as drug delivery system for sustained release of 5-fluorouracil. Mat. Sci. Eng. C Mater. 61, 158-168 (2016).

188. Piletska EV, Abd BH, Krakowiak AS et al. Magnetic high throughput screening system for the development of nano-sized molecularly imprinted polymers for controlled delivery of curcumin. Analyst 140(9), 3113-3120 (2015).

189. Zavareh S, Mahdi M, Erfanian S, Hashemi-Moghaddam H. Synthesis of polydopamine as a new and biocompatible coating of magnetic nanoparticles for delivery of doxorubicin in mouse breast adenocarcinoma. Cancer Chemother. Pharmacol. 78(5), 1073-1084 (2016).

190.Zaidi SA. Molecular imprinted polymers as drug delivery vehicles. Drug Deliv. 23(7), 2262-2271 (2016).

191. Ruela ALM, De Figueiredo EC, De Araújo MB, Carvalho FC, Pereira GR. Molecularly imprinted microparticles in lipid-based formulations for sustained release of donepezil. Eur. J. Pharm. Sci. 93, 114-122 (2016)

192. Chen H, Zhang W, Yang N, Chen C, Zhang M. Chitosan-based surface molecularly imprinted polymer microspheres for sustained release of sinomenine hydrochloride in aqueous media. Appl. Biochem. Biotechnol. 185(2), 370-384 (2018). 